\title{
La procédure civile internationale et la procédure civile transnationale: l'incidence de l'intégration économique régionale
}

Frédérique Ferrand

\section{Introduction}

1. De plus en plus, les auteurs parlent d'un "droit commun du procès ", ${ }^{1}$ qui ne se limiterait d'ailleurs pas à la seule procédure civile, mais engloberait également un certain nombre de principes de procédure devant les juridictions pénales et administratives. Ce «droit processuel horizontal" en ce sens qu'il apparaît aujourd'hui un tronc commun de règles portées par l'influence prépondérante des instruments internationaux des droits de l'homme, ne se construit pas uniquement au niveau mondial. Certes, le pacte international relatif aux droits civils et politiques dit également Pacte de New York du 19 décembre $1966^{2}$ et la jurisprudence du Comité des droits de l'homme $e^{3}$ ont contribuéà répandre dans le mon-

1 V. notamment S. Guinchard et autres, Droit processuel $N^{\circ} 59$ Dalloz éd., Paris, 2000 .

2 Ce pacte a été précédé de la Déclaration universelle des droits de l'homme du 10 décembre 1948 , qui contient dans ses articles $8^{\circ}$ à $11^{\circ}$ un certain nombre de garanties procédurales : droit à un recours effectif devant les juridictions en cas de violation des droits fondamentaux, interdiction des arrestations et des détentions arbitraires, droit à un procès équitable, présomption d'innocence, non rétroactivité des lois pénales.

3 Ce Comité peut, en vertu du protocole facultatif du 19 décembre 1966, connaître de recours individuels formés par des particuliers contre des Etats qui ont accepté cette possibilité ; le requérant fait alors une " communication " au Comité, dans laquelle il indique en quoi ses droits fondamentaux ont été violés. Le Comité se prononce d'abord sur la recevabilité de la communication, puis recueille les observations des parties et rend des " constatations " (et non pas des jugements, car le Comité n'a pas le statut de juridiction) qui n'ont pas de force obligatoire. 
de entier des valeurs fondamentales qui concernent en partie les garanties de procédure; ces dernières sont ancrées dans l'article $14^{\circ}$ du Pacte, consacré au procès équitable (égalité des justiciables devant les tribunaux, droit à ce que la cause soit entendue équitablement et publiquement par un tribunal compétent, indépendant et impartial, établi par la loi).

2. Ainsi, certains n'ont pas hésité à parler d'une " chaîne du droit du procès à travers le monde " ${ }^{4}$ ou de "modèle universel de procès " Mais cette chaîne ne se construit pas uniquement sur le plan mondial, à l'aide d'instruments d'intégration dépassant le champ d'un continent. En effet, il existe des ensembles régionaux qui, par le biais d'une intégration économique ou même politique, sont parvenus à harmoniser, voir à unifier un certain nombre de domaines du droit du procès. Un des premiers ensembles est bien entendu l'Europe, qui présente deux visages: celui du Conseil de l'Europe regroupant quarante quatre Etats autour de la Convention européenne des droits de l'homme et des libertés fondamentales du 4 novembre $1950,{ }^{6}$ et celui de l'Union européenne, qui devient productrice de normes de procédure ${ }^{7}$ et de droit international privé de plus en plus nombreuses en raison de l'évolution des textes qui la gouvernent et que nous retracerons au cours de ce rapport.

3. Hormis l'Europe, d'autres droits processuels régionaux existent, fondés toujours sur la prépondérance de la notion de droits et libertés fondamentaux: la Convention américaine relative aux droits de l'homme adoptée le 22 novembre 1969 par les Etats membres de l'Organisation des Etats américains à San José, et qui est entrée en vigueur le 18 juillet 1978. Alors que les Etats-Unis n’ont toujours pas ratifié ce texte fédérateur,

4 S. Guinchard, op. cit., $N^{\circ} 59$.

5 S. Guinchard, op. cit., $N^{\circ} 57$.

$6 \mathrm{~V}$. l'exemple de la Lituanie qui a adhéré à la Convention en 1995 et a depuis lors modifié son Code civil et son Code de procédure civile ; la réforme de ce dernier tendait à mieux harmoniser les rôles respectifs des parties et du juge dans le procès civil, à assurer la concentration de la procédure dans un but de célérité de la justice -objectif d'ailleurs imposé par l'art. $6,1^{\circ} \mathrm{CEDH}$ - à rendre conformes les règles internes de procédure aux traités internationaux ratifiés par la Lituanie. V.V. Mizaras et V. Nekrosius, «Das neue Zivilund Zivilprozessrecht" in: Litauen, ZEuP2002, p. 466 et notamment p. 480 et s.

7 LUnion européenne peut également susciter des réformes de procédure nationales au sein de certains Etats membres, qui désirent aligner certaines règles de procédure sur le droit de la majorité d'autres Etats membres, v. par exemple la réforme du Code espagnol de procédure civile, J. Fröhlingsdorf et K.H. Lincke, "Das neue spanische Zivilprozessgesetz» (LEC), in : RIW 2001, p. 357. 
vingt-cinq Etats d'Amérique du Sud et d'Amérique centrale ont procédé à une telle ratification ${ }^{8}$. En Afrique également a été adoptée le 27 juin 1981 la Charte africaine des droits de l'homme et des peuples, qui est entrée en vigueur le 21 octobre 1986. ' Le Protocole de Ouagadougou, signé le 9 juin 1998, instituera dès son entrée en vigueur ${ }^{10}$ une Cour africaine des droits de l'homme et des peuples qui pourra être saisie par les individus et les organisations non gouvernementales, et dont les arrêts auront l'autorité de la chose jugée. La transformation de l'Organisation de l'Unité Africaine (OUA) en Union africaine, sur le modèle de l'Union européenne, annoncée à Durban en juillet 2002, devrait entraîner des modifications dans le sens d'une plus grande intégration régionale.

En revanche, l'Asie et l'Océanie ne se sont engagées dans aucun processus d'harmonisation régionale, de garantie commune des droits et libertés fondamentaux de fond ou de procédure.

Tout l'existant en matière de droits de l'homme contient en partie une connotation processuelle qui ne se limite pas aux garanties en matière pénale, mais s'étend au procès civil. C'est pourquoi il paraît aujourd'hui impossible de parler de procédure civile internationale ou transnationale sans faire état des sources relatives aux droits de l'homme.

4. Est-on pour autant en route vers un droit processuel unitaire? Et cela serait-il d'ailleurs souhaitable? Les instruments précédemment cités sont à la fois grands et modestes. Grands par l'idéal qu'ils portent et proclament. Modestes car ils ne concernent pas l'ensemble du champ de la procédure, mais seulement cette dernière prise sous l'angle des garanties fondamentales en vue d'un procès équitable, maittre mot aujourd'hui. Equité, égalité des armes, non discrimination, voici les mots clés de la procédure mondiale à venir.

8 V. S. Guinchard, op. cit., $\mathrm{N}^{\circ} 60$. Ce texte crée une Commission et une Cour interaméricaine des droits de l'homme, et un droit de recours des individus et des Etats est établi.

9 Cette Charte a été adoptée à Nairobi par la Conférence de l'Organisation de l'Unité africaine. Le contrôle de l'application de la Charte est toutefois réservé à la Commission africaine des droits de l'homme, et aucun droit de recours individuel n'est prévu. La Commission qui se voit adresser une pétition individuelle, ne peut s'en saisir que si la majorité absolue de ses membres le requiert. En outre, c'est la Conférence de l'Organisation de l'Unité africaine, organisme intergouvernemental, qui est chargée de suivre ou non les recommandations de la Commission; cette dernière ne peut en aucun cas transmettre directement ses remarques à l'Etat concerné par un manquement.

10 Cette entrée en vigueur suppose quinze ratifications des Etats signataires. 
5. Parallèlement à l'avènement d'une "justice des droits de l'homme", dénommés aussi droits et libertés fondementaux, sont apparus dans le monde divers modèles d'intégration économique, qui ont en partie conduit à des intégrations juridiques en matière de procédure civile. Peuvent être cités là l'ALENA, le MERCOSUR ${ }^{11}$ ou encore la Communauté européenne devenue Union européenne. C'est ainsi que par exemple s'est réuni, sur le plan européen, un groupe d'experts qui, il y a quelques années, a travaillé sur les règles de procédure civile des Etats membres afin de proposer certains principes qui auraient pu être facteurs d'harmonisation (Commission Storme). ${ }^{12}$

6. Longtemps, des réticences ${ }^{13}$ ont été exprimées envers une harmonisation de la procédure civile au motif que les traditions nationales en la matière seraient irréductibles, ${ }^{14}$ comme cela est parfois ${ }^{15}$ encore affirmé à propos du droit de la famille (à l'exception éventuelle du droit des régimes matrimoniaux). ${ }^{16}$ On a parfois aussi évoqué le lien fort qui

11 V. par exemple la Convention de 1994 unissant les Etats du MERCOSUR en matière de compétence judiciaire, de reconnaissance et d'exécution: Protocole de Buenos Aires sur la compétence internationale dans les litiges en matière contractuelle. Pour une version allemande de ce texte, v. IPRax, 1999, p. 127.

12 V. Rapprochement du droit judiciaire dans l'Union européenne, Storme éd., Dordrecht, 1994. V. aussi G. Walter et F. Walther, «International Litigation : Past Experiences and Future Perspectives" in: Essays on Transnational and Comparative Civil Procedure, sous la dir. de F. Carpi et M. A. Lupoi, Giappichelli éd., Turin, 2001, p. 183 et s.

13 Retraçant certaines de ces réticences, v. P. Biavati, «Is Flexibility a Way to the Harmonization of Civil Procedural Law in Europe?» in: Essays on Transnational and Comparative Civil Procedure, sous la dir. De F. Carpi et M.A. Lupoi, Giappichelli éd., Turin, 2001 , p. 85 et notamment p. 90 et s.

14 V. avant-propos de G. Hazard, R. Stürner, M. Taruffo et A. Gidi au Projet de Principes et Règles transnationaux de procédure civile (Joint American Law Institue / Unidroit Working Group on Principles and Rules of Transnationale Civil Procedure), Rome, mai, 2002, p. 1.

15 V. H. Kronke, Ziele - Methoden, Kosten - Nutzen: Perspektiven der Privatrechtsharmonisierung nach 75 Jahren UNIDROIT, JZ 2001, p. 1149 et plus spécialement p. 1156.

16 Pourtant, la Commission commence à formuler diverses propositions en matière de droit de la famille; un groupe de travail d'universitaires s'est en outre constitué en septembre 2001, la Commission de droit européen de droit de la famille (Commission on European Family Law), dont l'objectif est de traiter - à partir de questionnaires remplis par un spécialiste de chaque pays retenu - de divers thèmes de droit de la famille et de proposer des principes communs pour tous les Etats d'Europe. Le premier thème de réflexion choisi est les causes de divorce et la prestation compensatoire ou pension alimentaire entre époux après divorce. 
existerait entre les règles de procédure civile et d'organisation juridictionnelle et les Constitutions nationales. Ces temps sont dépassés, et il apparaît tant sur le plan régional lorsqu'existe une intégration économique (comme en Europe par exemple, où encore plus depuis le Traité d'Amsterdam, il est admis qu'une certaine harmonisation de la procédure civile peut être nécessaire pour la réalisation des objectifs du Traité, notamment l'objectif de libre circulation des personnes, ${ }^{17} \mathrm{v}$. Art. $65^{\circ}$ ), que sur le plan mondial, que des souhaits de rapprochement se font jour en droit processuel. Ces souhaits se fondent en général sur la volonté de non discrimination entre parties nationales et étrangères dans le procès, également sur la prévisibilité souhaitée du déroulement de la procédure, qui permet de réaliser en partie l'égalité des armes entre les protagonistes s'opposant dans le litige. ${ }^{18}$

7. Le sujet qui m'est imparti est immense; son étendue rend difficile un traitement exhaustif. En outre, les différents participants à la table ronde qui suivra ce rapport introductif auront certainement de riches apports à faire, notamment au sujet de continents ou de pays qui me sont moins familiers que l'Europe.

8. Procédure internationale et procédure transnationale prennent parfois appui sur des réalisations préexistantes dans le cadre d'institutions régionales. Parfois au contraire, elles s'en abstraient pour diverses raisons qu'il conviendra d'évoquer. ${ }^{19}$ Le sujet sera traité en trois points successifs: tout d'abord l'examen de l'adoption de règles de procédure civile au sein d'un modèle d'intégration régionale poussée à l'aide de l'exemple de l'Europe, qui se réunit à la fois au sein du Conseil de l'Europe et, dans un

17 V. la formule de l'article 65 Traité CE : "dans la mesure nécessaire au bon fonctionnement du marché intérieum, qui inclut l'objectif de libre circulation des personnes. Toutes les mesures en matière civile contenues dans le Titre IV du Traité CE sont liées à la libre circulation des personnes, qui constitue un des éléments du marché intérieur dans la définition qu'en donne l'Art. $14,2^{\circ}$ Traité $\mathrm{CE}$, dans le même sens, J. Basedow, The Communitarization of the Conflict of Laws under the Treaty of Amsterdam, CMLR 37 (2000), p. 687 et plus spécialement p. 701.

18 Sur le besoin d'une harmonisation par des règles transnationales de procédure, v. le rapport de G. Hazard, R. Stürner, M. Taruffo et A. Gidi précédant le projer ALIUNIDROIT, op. cit., p. 2 et s. qui sera traité plus loin dans cet article.

$19 \mathrm{~V}$. par exemple le projet de Convention mondiale sur la compétence judiciaire et les jugements négocié dans le cadre de la Conférence de La Haye de droit international privé, infra. 
cadre plus réduit, dans l'Union européenne (I). Ensuite sera abordé un modèle d'intégration mondiale non pas encore réalisé, mais recherché sous deux formes de projet: celui de la Conférence de La Haye de droit international privé d'une convention mondiale en matière de compétence judiciaire, de reconnaissance et d'exécution, et le projet de l'American Law Institute et d'UNIDROIT de Principes et règles transnationaux de procédure civile (II). Enfin sera posée en troisième partie la question du modèle préférable et des éventuelles interactions entre ces deux types d'intégration: l'existence de l'un nuit-elle à l'autre ou au contraire s'enrichissent-ils mutuellement? Une intégration mondiale est-elle possible et même souhaitable sans préalable régional?; le préalable régional peut-il même se révéler un obstacle à une intégration mondiale? Ces diverses questions seront illustrées à l'aide de la procédure civile européenne et internationale (III).

\section{Procédure civile et modèle d'intégration régionale poussé: l'exemple de l'Europe}

9. L'intégration régionale européenne comprend deux dimensions: celle du Conseil de l'Europe et des droits de l'homme, avec la Convention européenne de sauvegarde des droits de l'homme et des libertés fondamentales du 4 novembre 1950 qui, appliquée par la Cour européenne des droits de l'homme de Strasbourg, a connu une interprétation extensive des garanties processuelles qu'elle contient, notamment dans l'article $6^{\circ}$. Il peut être affirmé aujourd'hui que l'interprétation jurisprudentielle de la Convention a conduit à une certaine harmonisation du droit des Etats signataires en matière de garanties du procès, le mot-clé étant celui de "procès équitable» (fair trial, faires Verfahren). Les Etats membres du Conseil de l'Europe sont actuellement au nombre de quarante quatre; tous les Etats de l'Union européenne en font partie et sont ainsi liés depuis de nombreuses années par des garanties processuelles fondamentales (1) qui ont largement précédé l'intervention de l'Union européenne en matière de procédure civile (2).

\section{Au sein du Conseil de l'Europe.}

10. Le cadre de cette étude ne permet pas d'entrer dans le détail des réalisations processuelles issues de la Convention européenne des droits 
de l'homme du 4 novembre 1950 et des exigences de son article $6{ }^{\circ} .{ }^{20} \mathrm{Ce}$ dernier, qui dispose que "toute personne a droit à ce que sa cause soit entendue équitablement, publiquement et dans un délai raisonnable, par un tribunal indépendant et impartial, établi par la loi, qui décidera, soit des contestations sur ses droits et obligations de caractère civil, soit du bien fondé de toute accusation en matière pénale dirigée contre elle», ${ }^{21}$ est considéré comme la pierre angulaire du droit processuel européen. Il contient, dans l'interprétation extensive qui en a été faite par la Cour européenne des droits de l'homme de Strasbourg, toutes les garanties essentielles du procès qui peuvent être résumées sous l'intitulé Equité de la procédure. Le contenu de l'article $6^{\circ}$ est pratiquement repris mot pour mot par la Charte des droits fondamentaux de l'Union européenne adoptée par le Conseil européen de Nice le 7 décembre 2000; cette Charte consacre un certain nombre de principes fondamentaux de procédure tels que le droit à un recours effectif et celui d'accéder à un tribunal impartial, le droit général à un procès équitable (v. Art. $47^{\circ}, \$ 2$ qui reprend en substance le contenu de l'article $\left.6^{\circ} \mathrm{CEDH}\right) .{ }^{22}$

20 Sur l'article $6^{\circ}$ et le procès civil, notamment français, v. F. Ferrand, "I diritti fondamentali e il processo civile in Francia", in : Dirittifondamentali e giustizia civile in Europa, éd. Giappichelli, Turin, 2002, publication faisant suite au , Colloque de Taormina 31 mai- 2 juin 2001 dans le cadre de l'association italienne de droit comparé; Le procès civil français et la Convention européenne des Droits de l'Homme, Bulletin de la Société de Législation comparée 1996 ; Der franzözische Zivilprozeß im Lichte von Art. 6 Abs. 1 EMRK, in : Festschrift Henrich, Gieseking éd., Bielefeld, 2000, p. 133.

21 L'article ajoute que "le jugement doit être rendu publiquement, mais l'accès de la salle d'audience peut être interdit à la presse et au public pendant la totalité ou une partie du procès dans l'intérêt de la moralité, de l'ordre public ou de la sécurité nationale dans une société démocratique, lorsque les intérêts des mineurs ou la protection de la vie privée des parties au procès l'exigent, ou dans la mesure jugée strictement nécessaire par le tribunal, lorsque dans des circonstances spéciales, la publicité serait de nature à porter atteinte aux intérêts de la justice».

22 La Charte n'a pas encore force obligatoire envers les Etats membres. En matière processuelle, on peut s'interroger sur son utilité, du fait que son contenu recouvre largement celui de l'article $6^{\circ}$ et de l'article $13^{\circ} \mathrm{CEDH}$. La prolifération des instruments internationaux ou supra-nationaux de protection des droits de l'homme n'est pas forcément une garantie de meilleure justice, tant l'enchevêtrement des textes rend difficile leur llecture et l'appréciation de leur portée respective. En outre, les Etats membres de l'Union européenne ont tous adhéré à la Convention européenne des droits de l'homme et appliquent ses exigences processuelles comme droit directement applicable. 
11. La Convention européenne des droits de l'homme de 1950 constitue un ciment entre le droit des Etats signataires. Elle impose dans un premier temps que soit assuré un accès au tribunal, accès qui doit être effectif. Une fois cet accès consacré, il importe que le procès se déroule devant une juridiction indépendante et impartiale établie par la loi (principe du juge naturel). Le déroulement du procès doit être équitable, ce qui suppose, pour faciliter une possibilité de contrôle, une publicité des débats et une égalité des armes entre les parties, de même qu'une information des parties sur les motifs de la décision rendue. La décision doit en outre être rendue et pouvoir être exécutée dans un délai raisonnable. Dans toutes ces exigences, on peut apercevoir la naissance d'un «modèle de procès équitable européen" et d'un "ordre public démocratique européen". ${ }^{23}$

12. La détermination du tribunal compétent doit se faire selon des règles garantissant le droit au juge naturel: nul ne peut être soustrait à son juge naturel en ce sens que la loi doit prédéterminer ou permettre la prédétermination de la juridiction compétente afin d'éviter des manipulations de compétence suivant le litige ou l'identité de ses protagonistes. Les dispositions législatives sur la compétence sont, dans ce contexte, davantage établies pour la protection du justiciable contre l'arbitraire que pour son information et la prévisibilité de la procédure. Le droit national doit en outre garantir un accès effectif au tribunal, concept qui a donné lieu à une jurisprudence «musclée» de la Cour de Strasbourg, ${ }^{24}$ l'accès effectif au tribunal peut nécessiter l'octroi par l'Etat, d'une aide juridictionnel au plaideur impécunieux, comme l'admet également la proposition de la Commission européenne en matière d'aide judiciaire. ${ }^{25}$

23 R. De Gouttes, "La Convention européenne des Droits de l'Homme et le juge français», RIDC 1999, p. 7. V.aussi A. -M. Gouron-Mazel, "La Cour de cassation française face à la Convention européenne des Droits de l'Hommen, JCP 1996. I. 3937 ; Convention européenne des Droits de l'Homme, application par le juge français, 10 ans de jurisprudence, Litec éd., Paris, 1998. Comp. S. Guinchard, «Linfluence de la Convention européenne des Droits de l'Homme et de la jurisprudence de la Cour européenne sur la procédure civile», Gaz. Pal. 27 au 31 août 1999, p. 2.

24 V. notamment l'arrêt précurseur C. eur. dr. h., 7 oct. 1979, Airey c/ Irlande, Série A, No32. Pour un rappel récent, C. eur. dr. h., 19 sept. 2000, Gnahorté cl France, D. 2001. 725 , note F. Rolin.

25 Proposition de directive de la Commission sur l'amélioration de l'accès à la justice dans les affaires transfrontalières par l'établissement de règles minimales communes relatives à l'aide judiciaire et à d'autres aspects financiers des procédures civiles, COM (2002), 13 final, disponible sur le site internet europa.eu.int. 
Là encore, les deux pôles européens - Conseil de l'Europe et Union européenne- qui édictent des normes ou proposent des Conventions pour des Etats destinataires partiellement semblables, se rencontrent dans l'affirmation de garanties fondamentales du procès.

13. Une fois l'accès au tribunal réellement ouvert, le procès bénéficie à son tour d'un certain nombre de principes de procédure: indépendance et impartialité des juges sont requis comme conditions d'un procès équitable. Ce dernier suppose également, pour sa garantie, une procédure publique en principe, ainsi que le prononcé en public du jugement, qui doit être motivé afin que les plaideurs puissent contrôler que les juges ont bien répondu à toutes leurs conclusions. De façon générale, la procédure doit respecter les droits de la défense, et le principe de la contradiction, qui est un des aspects de la notion d'égalité des armes (Waffengleichheit) dans le procès. Cette notion d'égalité des armes a introduit de nombreux chamboulements dans le droit de certains Etats signataires, qui ont dû rompre avec un certain nombre de pratiques, telles que celle de la Cour de cassation belge ${ }^{26}$ ou française par exemple de donner au ministère public la parole en dernier, juste avant le délibéré, si bien que les prévenus ou les parties ne peuvent pas répondre. ${ }^{27}$ Par son rôle interprétatif et unificateur, la Cour européenne des droits de l'homme a ainsi fait progresser de nombreux aspects du droit processuel national des Etats signataires.

14. Enfin, le procès équitable suppose un jugement rendu dans un délai raisonnable et susceptible d'exécution ${ }^{28}$ dans un tel délai. Il est bien connu que la grande majorité des arrêts de condamnation rendus à Strasbourg dont fondés sur cette exigence, notamment les décisions concernant l'Italie, mais également la France. ${ }^{29}$ L'idée sous-tendant

26 C. eur. dr. h., 20 février 1996, Vermeulen c/ Belgique, Rec. 1996. I. 224 ; 25 juin 1996, Van Orshoven cl Belgique, Rec. 1997. III. 1039.

27 V. C. eur. dr. h., 31 mars 1998, Reinhard et Slimane Kaïd c/ France, RTDCiv. 1998, p. 511 , obs. J.-P. Marguénaud. La Cour de cassation française a introduit depuis une nouvelle pratique : celle selon laquelle les parties ou le prévenu peuvent faire parvenir à la Cour des notes en délibéré répondant au ministère public, si vien que ce dernier n'a pas en fait le dernier mot. Cette pratique est expressément approuvée par la Cour de Strasbourg, mais elle n'avait pas encore été suivie dans l'affaire Slimane Kaïd.

28 V. C. eur. dr. h., 19 mars 1997, Horsby c/ Grèce, AJDA 1997. 9867, obs. J.-F. Flauss ; JCP 1997.II. 22 949, note O. Dugrip et F. Sudre.

$2960 \%$ du contentieux français devant les instances de Strasbourg jusqu'au 30 décembre 1998 concernait des questions de durée de la procédure. Ce taux est largement plus élevé encore pour l'Italie. 
l'obligation de célérité de la justice est qu'une justice lente peut équivaloir à un déni de justice. La Cour de Strasbourg a développé une jurisprudence au cas par cas en établissant un certain nombre de critères permettant d'apprécier si la durée de la procédure a été excessive ou pas et justifie une constatation de violation de l'Art. $6^{\circ}, 1$ : complexité de l'affaire, comportement des parties, diligence du tribunal et des autorités judiciaires de façon générale; parfois aussi, l'importance de l'affaire pour le requérant peut être prise en compte.

15. La Convention européenne de sauvegarde des droits de l'homme a fourni une contribution remarquable à la délimitation des contours du procès équitable. Elle a un rôle unificateur ou en tout cas d'harmonisation du droit national des Etats signataires selon des standards minimum que la Cour de Strasbourg a élevés avec sa jurisprudence progressive et exigeante. Il s'agit donc là d'un instrument international dont le succès est à saluer. La question s'est toutefois posée à la suite de l'intervention de la Cour de Justice des Communautés en matière de droits fondamentaux, et en outre depuis l'adoption par l'Union européenne, d'une Charte des droits fondamentaux, de savoir si la jurisprudence de la Cour de Strasbourg garderait toute sa portée, où si ne coexisteraient pas parallèlement des jurisprudences européenne d'une part (Cour européenne des droits de l'homme), communautaire de l'autre (Cour de Justice des Communautés) en matière de droits fondamentaux, ${ }^{30}$ ce qui risquerait de conduire à des contradictions regrettables pour la prévisibilité juridique et pour l'harmonie des droits processuels fondamentaux. ${ }^{31} \mathrm{La}$ Cour de Justice des Communautés s'est jusqu'à présent largement fondée sur la Convention européenne des droits de l'homme et les traditions constitutionnelles des Etats membres pour protéger un certain nombre de droits et libertés. Mais lorsque la Charte européene aura acquis force obligatoire, la Cour de Justice en sera la gardienne et il n'est pas exclu que certaines dispositions, similaires à celles de la Convention européenne de 1950, soient interprétées différemment, ${ }^{32}$ ce qui nuirait à un système cohérent de protection. ${ }^{33}$

$30 \mathrm{La}$ Cour de Justice des Communautés s'est en effet prononcée contre l'adhésion de l'Union européenne à la Convention européenne des droits de l'homme du 4 novembre 1950. L'Union européenne n'est pas non plus membre du Conseil de l'Europe.

31 Sur cette question, v. J. Limbach, «Das Bundesverfassungsgericht und der Grundrechtsschutz in Europa», in : NJW 2001, p. 2913 et notamment p. 2918.

32 Pour des exemples de divergences d'ores et déjà constatées entre les jurisprudences de la Cour européenne des droits de l'homme et de la Cour de Justice des Communautés, v. Albert et Widmaier, $E u G R Z 2000$, p. 497 et spécialement p. 503 et s. 
Des mécanismes d'échange d'information et un souci d'unifier les droits fondamentaux de procédure dans l'Union européenne et le Conseil de l'Europe, et ce dans l'intérêt des citoyens, devraient favoriser des jurisprudences largement convergentes.

16. Parallèlement à la construction d'un droit processuel européen issu des droits et libertés fondamentaux, se construit, à une échelle plus réduite, celle de l'Union européenne, une intégration juridique extrêmement rapide et étendue qui, depuis peu, touche directement le droit international privé et la procédure civile des Etats membres.

\section{Au sein de l'Union européenne.}

17. Nous avons déjà souligné dans un autre contexte ${ }^{34}$ l'extension considérable de ses pouvoirs législatifs qu'a connue la Communauté européenne ces dernières années. Rappelons que le Traité sur l'Union europénne, dit Traité de Maastricht de 1992, avait introduit dans le troisième pilier (domaine relevant de la négociation intergouvernementale entre les Etats membres) la coopération judiciaire en matière civile. Celleci, par le Traité d'Amsterdam de 1997 entré en vigueur le ler mai 1999, a ensuite été placée dans le premier pilier (article $65^{\circ} \mathrm{du}$ Traité $\mathrm{CE}$ ), ce qui a transféré la compétence législative aux institutions communautaires. ${ }^{35}$ L'action de la Commission a parfois été qualifiée de bureaucratique, impatiente, caractérisée par une absence d'égards envers les Etats membres; ${ }^{36}$ le principe de subsidiarité communautaire ${ }^{37}$ contenu dans l'article $5,2^{\circ}$

33 J. Limbach, préc., NJW2001, p. 2918.

34 V. supra $\mathrm{n}^{\circ} 6$.

35 V. D. Besse, Die justizielle Zusammenarbeit in Zivilsachen nach dem Vertrag von Amsterdam und das EuGVÜ, ZEuP 1999, p. 107. L'auteur insiste sur le fait qu'au bout de cinq ans, c'est-à-dire en 2004, le monopole d'initiative des mesures fondées sur l'article $65^{\circ}$ TraitéCE reviendra à la Commission, alors que pour l'instant les Etats membres ont également un droit de proposition. L'auteur parle des conséquences «énormes » des modifications apportées par le Traité d'Amsterdam sur la coopération judiciaire en matière civile.

36 V. H. Schack, "Die Kommission auf dem Holzweg von Amsterdam», in: ZEuP 1999 , p. 805 et s.

37 V. P. Biavati, «Is Flexibility a Way to the Harmonization of Civil Procedural Law in Europe ?», in : Essais on Transnational and Comparative Civil Procedure, sous la direction de F. Carpi et M. A. Lupoi, Giappichelli éd., Turin, 2001, p. 85 et plus spécialement p. 87 ets. 


\section{du Traité serait peu respecté..$^{38}$}

18. La production de normes communautaires en matière de procédure civile s'est considérablement accrue avec l'entrée en vigueur du traité d'Amsterdam du 2 octobre 1997: outre le règlement CE N ${ }^{\circ}$ 1347/2000 du 29 mai 2000 relatif à la compétence, la reconnaissance et l'exécution des décisions en matière matrimoniale et en matière de responsabilité parentale des enfants communs ${ }^{39}$ et celui $\mathrm{N}^{\circ} 44 / 2001$ du 22 décembre 2000 remplaçant la Convention de Bruxelles ${ }^{40}$ et relatif à la compétence judiciaire, à la reconnaissance et à l'exécution des décisions en matière civile et commerciale, ${ }^{41}$ on peut également citer le règlement $C E \mathrm{~N}^{\circ} 1348 /$ 2000 du 29 mai 2000 sur la signification et la notification dans les Etats membres des actes judiciaires et extrajudiciaires en matière civile et

38 Ibid . Lauteur parle de la " rabiate Rechtsvereinheitlichungspolitik von oben » de la Commission européenne.

39 JO CE L 160 du 30 juin 2000, p. 19.

40 Un des avantages de la transformation de la Convention de Bruxelles en règlement européen est notamment l'absence de nécessité désormais de ratification par les Etats membres de toute modification de convention, comme cela était au contraire indispensable chaque fois qu'un nouvel Etat devenait membre de la Communauté et qu'une convention étendait l'application de la Convention de Bruxelles à son territoire (c'est ainsi par exemple que la Belgique n'a ratifié la Convention de San Sebastian de 1989 qu'en 1997, si bien que la Convention n'a pas pu s'appliquer entre la Belgique d'une part, l'Espagne et le Portugal d'autre part, pendant plus de huit ans).

41 JO CE L 12 du 16 janvier 2001, p. 1. Parmi les nombreux commentaires parus sur ce règlement, v. J.-P. Beraudo, Le Règlement (CE) du Conseil du 22 décembre 2000 concernant la compétence judiciaire, la reconnaissance et l'exécution des décisions en matière civile et commerciale, JDI2001, p. 1033 ; Ch. Bruneau, "Les règles européennes de compétence en matière civile et commerciale», JCP éd.G 2001. I. 304 ; G. Droz et H. Gaudemet-Tallon, La transformation de la Convention de Bruxelles du 27 septembre 1968 en Règlement du Conseil concernant la compétence judiciaire, la reconnaissance et l'exécution des décisions en matière civile et commerciale, Rev. crit. DIP 2001, p. 601. Sur l'absence de possibilité pourtant de fonder ce règlement sur l'article $65^{\circ}$ Traité $\mathrm{CE}$, v. H. Schack, Die Kommission auf dem Holzweg von Amsterdam, ZEuP 1999, p. 807, qui considère que les mesures fondées sur cet article doivent concerner la libre circulation des personnes, ce qui ne serait pas le cas pour la libre circulation des jugements, que les mesures doivent se limiter à ce qui est nécessaire au bon fonctionnement du marché intérieur (ce qui exclurait le droit de la famille), et enfin que l'article 65 ne peut atteindre un but d'unification juridique du fait que ni le Royaume-Uni, ni l'Irlande, ni le Danemark ne sont a priori parties aux mesures édictées sur son fondement (sauf, pour le Royaume-Uni et l'Irlande, s'ils demandent expressément à être associés à ces mesures). 
commerciale, ${ }^{42}$ le règlement $C E N^{\circ} 1346 / 2000$ du 29 mai 2000 relatif aux procédures d'insolvabilitée $e^{43}$ ou encore le règlement CE $N^{\circ} 1206 /$ 2001 du 28 mai 2001 relatif à la coopération entre les juridictions des Etats membres dans le domaine de l'obtention des preuves en matière civile et commerciale. ${ }^{44}$ Doit également être mentionné le règlement $\mathrm{CE}$ $\mathrm{N}^{\circ} 743 / 2002$ du 25 avril $2002^{45}$ établissant un cadre général communautaire en vue de faciliter la coopération judiciaire en matière civile. $^{46}$

42 JO CE L 160 du 30 juin 2000, p. 37.

43 JO CE L 160 du 30 juin 2000, p. 1. V. notamment M-E. Mathieu-Bouyssou, Aperçu des règles de droit judiciaire privé relatives aux procédures d'insolvabilité européennes après le règlement communautaire $\mathrm{N}^{\circ} 1346 / 2000, D .2002$.p. 2245.

44 JO CE L 174 du 27 juin 2001, p. 1.V. Ch. Berger, Die EG-Verordnung über die Zusammenarbeit der Gerichte auf dem Gebiet der Beweisaufnahme in Zivil- und Handelssachen, IPRax 2001, p. 522.

$45 \mathrm{JO}$ CE L 115 du $1^{\text {tr }}$ mai 2002, p. 1. Ce règlement donne un cadre aux activités que la Commission peut susciter et financer, activités telles que études, recherches, séminaires, conférences, réunions d'experts, publications, manuels, bases de données et/ou sites internet. Le règlement s'applique pour la période 2002-2006 ; il se donne comme objectif ambitieux d'encourager la coopération judiciaire en matière civile afin d'assurer la sécurité juridique et d'améliorer l'accès à la justice, de promouvoir la reconnaissance mutuelle des décisions judiciaires, de favoriser le rapprochement nécessaire des législations, d'éliminer les obstacles que créent les disparités en matière de droit civil et de procédure civile, d'améliorer la connaissance réciproque des systèmes juridiques et judiciaires des Etats membres, de permettre une application correcte des instruments communautaires et d'améliorer l'information du public sur l'accès à la justice, la coopération judiciaire et les systèmes juridiques des Etats membres en matière civile. Certains pays tiers peuvent se joindre à ce cadre général (Arr. $4^{\circ} \mathrm{du}$ règlement). Des actions de formation, d'échanges et stages, d'études et recherches, de rencontres et séminaires et une circulation d'informations sont prévues (Art. $5^{\circ}$ ). Le règlement contient des règles de financement par la Communauté ainsi qu'un contrôle de la Commission sur les actions financées. La Commission est assistée d'un Comité consultatif; elle doit au plus tard le 30 juin 2004 faire un rapport au Parlement européen et au Conseil sur la mise en œuvre du cadre général, et notamment sur les résultats du contrôle, les rapports et la surveillance des activités.

$46 \mathrm{~V}$. aussi la décision $\mathrm{N}^{\circ} 2001-470$ du 28 mai 2001 relative à la création d'un réseau judiciaire européen en matière civile et commerciale, JO CE L 174 du 27 juin 2001, p. 25. Ce réseau vise à faciliter la coopération judiciaire entre les Etats membres en matière civile et commerciale tant dans les domaines couverts par des instruments en vigueur que dans ceux où aucun instrument n'est encore applicable. Le réseau se compose de points de contacts désignés par chaque Etat membre, des instances et des autorités centrales communautaires, des magistrats de liaison et le cas échéant, de toute autre autorité judiciaire ou administrative ayant des responsabilités dans le domaine de la coopération judiciaire en 
19. Au cours de la conférence de Tampere de 1999, de nombreux domaines d'harmonisation avaient été envisagés: accès au droit, notamment dans le cadre de dénominateurs communs minimum en matière d'aide juridictionnelle, accélération des procédures juridictionnelles dans les litiges commerciaux et de la consommation, amélioration des modes alternatifs de règlement des litiges, ${ }^{47}$ facilitation de certains actes de procédure par standardisation de formulaires, principe de reconnaissance réciproque et d'exécution des décisions rendues dans un autre Etat membre sans même passer par une procédure d'exécution telle qu'elle existe dans la Convention de Bruxelles du 27 septembre 1968 et est maintenue, même si elle connaît un certain nombre d'allègements, dans le nouveau règlement $\mathrm{CE}$ du 22 décembre 2002 se substituant à la Convention de Bruxelles. La Conférence de Tampere a également recommandé une plus grande convergence des procédures civiles nationales, notamment dans les domaines des mesures

matière civile et commerciale. Le réseau devra également concevoir et tenir à jour un système d'information destiné au public, et vérifier l'application effective et concrète des actes communautaires ou des conventions en vigueur entre deux ou plusieurs Etats membres. Des réunions régulières auront lieu entre les points de contact (au moins une fois par semestre) afin notamment d'échanger leur expérience, de discuter les problèmes pratiques et juridiques rencontrés, d'identifier les meilleures pratiques à suivre dans le domaine de la coopération judiciaire...

47 A ce sujet, v. le livre vert rédigé par la Commission sur les modes alternatifs de résolution des conflits relevant du droit civil et commercial, COM (2002) 196 final, disponible sur le site internet europa.eu.int. Le sujer est "à la mode " et de nombreux Etats membres ont adopté des règles favorisant, voire imposant une conciliation ou médiation préalable au procès civil (v. par exemple la réforme allemande de juillet 2001 entrée en vigueur le $1^{\text {er }}$ janvier 2002 et le $\$ 278$ ZPO. Les techniques d'ADR (Alternative Dispute Resolution), connues en France sous les initiales MARC (Modes alternatifs de règlement des litiges) tendent à favoriser un meilleur accès à la justice, dont les tribunaux étatiques sont largement encombrés par une recrudescence d'actions en justice ; or l'accès à la justice est un droit fondamentale consacré tant par l'Art. $6^{\circ} \mathrm{CEDH}$ que par l'Art. $47^{\circ} \mathrm{de} \mathrm{la} \mathrm{Charte}$ des droits fondamentaux de l'Union européenne. Les ADR ou MARC sont des " instruments de la paix sociale" (v. livre vert de la Commission p. 9), leur approche consnsuelle augmente les chances des parties, une fois le conflit réglé, de pouvoir maintenir leur relations d'affaires. Rappelons que le Conseil de l'Europe a, dès 1998, adopté une recommandation sur la médiation familiale (http://cm.coe.int $/ \mathrm{ta} / \mathrm{rec} / 1998 / \mathrm{f} 98 \mathrm{r} 1$.htm $)$ et élabore actuellement un projet de recommandation sur la médiation civile (http:// www.legal.coe.int.civilandcommercial) ; la CNUDCI prépare également un loi modèle sur la conciliation en matière commerciale (http:// www.uncitral.org/fr-index.htm). 
provisoires, du droit de la preuve, de la procédure d'injonction de payer ${ }^{48}$ et des délais de procédure. ${ }^{49}$ Trois axes d'action prioritaires avaient donc été dégagés à Tempere : 1) un meilleure accès à la justice, 2) la reconnaissane mutuelle des décisions judiciaires et 3 ) une convergence accrue dans le domaine du droit procédural. ${ }^{50}$

20. La Commission européenne a d'ores et déjà proposé un certain nombre de textes à l'adoption du Conseil: tout d'abord, elle a présenté le 18 avril 2002 une proposition de création d'un titre exécutoire européen ${ }^{51}$ concernant les créances incontestées ${ }^{52}$ —ce qu'on appelle le "titre exécutoire européen ", qui serait " le projet pilote de suppression de l'exequatur-.$^{53}$

48 Pour une anticipation par l'Etat espagnol, qui a introduit dans son droit une procédure d'injonction de payer assez semblable à celle que connaît le droit allemand, v. J. Fröhingsdorf et K.H. Lincke, Das neue spanische Zivilprozesshrecht (LEC), RIW2001, p. 359.

49 V. B. HEb, Aktuelle Perspektiven der europäischen Prozessrechtsangleichung, $J Z$ 2001, p. 573.

50 Le sommet de Tempere a exprimé le souhait d'établir une meilleure collaboration entre les autorités des Etats membres afin de faciliter la vie des citoyens européens dont la mobilité est, par ailleurs encouragée par le développement du principe de la libre circulation. Les citoyens et opérateurs ne doivent pas être empêchés de faire valoir leurs droit en raison de l'incompatibilité des systèmes juridiques et administratifs des Etats membres. Il convient de régler les difficultés inhérentes à ces incompatibilités et complexités.

51 V. B. HEb, préc., JZ 2001, p. 578. V. aussi le texte Proposition de règlement du Conseil portant création d'un titre exécutoire européen pour les créances incontestées, document COM (2002) 159 final, disponible sur le site internet. europa.eu.int.

52 Le programme commun de la Commission et du Conseil relatifà des mesures de mise en œuvre du principe de reconnaissance mutuelle des décisions en matière civile et commerciale (JO C 12 du 15 janvier 2001, p. 1) adopté par le Conseil le 30 novembre 2000, a désigné la suppression de l'exequatur pour les créances incontestées comme l'une des priorités de la Communauté. Il est contradictoire que des décisions concernant des créances non contestées par le débiteur soient retardées dans leur exécution par la procédure d'exequatur; le recouvrement rapide des impayés est une nécessité absolue pour le commerce et représente une préoccupation constante des milieux économiques concernés par le bon fonctionnement du marché intérieur (exposé des motifs de la proposition de règlement, p. 1 et 2).

53 En ce sens les propos des ministres de la Justice de l'Union européenne réunis à Stockholm les 8 et 9 février 2001, et l'exposé des motifs de la proposition de règlement, $p$. 2. La Commission relève que l'expression titre exécutoire européen est souvent utilisée dans le sens d'une procédure uniforme d'obtention d'une décision qui serait alors exécutoire sans exequatur dans tous les Etats membres, mais que la mise en place d'une procédure uniforme et la suppression de l'exequatur sont des questions distinctes, la réponse à l'une n'étant pas un préalable nécessaire à l'autre. Elle a donc décidé, en matière de créances incontestées, de poursuivre les deux objectifs sans pour autant les regrouper dans un même instrument législatif. 
Ce texte rappelle que le principe de reconnaissance mutuelle des décisions judiciaires constitue la «pierre angulaire de la création d'un véritable espace judiciaire", qu'il convient, en matière de créances incontestées, de supprimer toutes les mesures intermédiaires à prendre avant l'exécution dans l'Etat membre où elle est demandée, ce qui permettra de se dispenser de l'intervention des autorités judiciaires d'un deuxième Etat membre ${ }^{54}$ avec les retard set les frais qui en résultent. ${ }^{55}$ Une référence est faite dans l'exposé des motifs à l'article $47^{\circ}$ de la nouvelle Charte des droits fondamentaux de l'Union européenne et à l'exigence posée par cet article d'assurer le plein respect du droit à accéder à un tribunal impartial. La proposition de règlement se divise en neuf chapitres (chapitre I : objet, champ d'application et définitions); chapitre II: le titre exécutoire européen ; chapitre III : normes minimales applicables aux procédures relatives aux créances incontestées; chapitre IV : exécution; chapitre V : transactions judiciaires et actes authentiques; chapitre VI: dispositions générales; chapitre VII : disposition transitoire; chapitre VIII: relation avec d'autres instruments (notamment avec le règlement $\mathrm{CE} \mathrm{N}^{\circ} 44 / 2^{\circ} 1$ du 22 décembre

54 Pour des critiques sur la suppression, en l'état actuel du droit national des Etats membres, de toute vérification avant que la décision d'un Etat membre puisse être rendue exécutoire sur le territoire d'un autre Etat memb, v. Ch. Kohler, «Systemwechsel im europäischen Anerkkennungsrecht : Von der EuGVVO zur Abschaffung des Exequaturs», in : Systemwechsel im europäischen Kollisionsrecht, Beck éd., Munich, 2002, p. 147 et spécialement, p. 158 et $s$. L'auteur relève notamment que le droit constitutionnel d'un certain nombre d' Etats membres s'oppose à une telle suppression de l'exequatur, par exemple celui de l'Allemagne, v. la décision de la C. const. fédérale de Karlsruhe du 22 mars 1983 (BVerfGE 63, p. 343 et spéc. p. 366) qui considère qu'il existe des limites constitutionnelles à l'introduction d'une possibilité générale d'exécution (directe), des titres exécutoires étrangers; elle ne serait possible que si les normes matérielles appliquées au soutien de la décision étrangère n'étaient pas contraires à l'ordre public de la République fédérale et si la procédure répondait à un standard minimum d'Etat de droit. Il ne serait pas exclu que la Cour constitutionnelle allemande se prononce à nouveau de cette façon face à un titre exécutoire européen, sauf bien entendu si la justice au sein des Etats membres était placée sous un "toit constitutionnel commun" et si une juridiction suprême surveillait l'exercice du pouvoir judiciaire (ibid., p. 161). Ch. Kohler considère en outre que la vision par la Communauté de ses pouvoirs en matière de coopération judiciaire civile et le programme de mesures annoncé sont incompatibles avec le principe d'habilitation limitée de la Communauté retenu par la Cour de Justice des Communautés dans l'arrêt Allemagne c/ Conseil et Parlement du 5 octobre 2000, affaire C-376/00, Rec. 2000. 1.8419.

55 En outre, la traduction sera bien souvent inutile, car des formulaires types multilingues seront employés pour le certificat de titre exécutoire européen. 
2000 qui s'est substitué à la Convention de Bruxelles de 1968); chapitre IX: dispositions finales)..$^{56}$

21. Une deuxième proposition de la Commission de directive (et non de règlement) du Conseil porte sur l'amélioration de l'accès à la justice dans les affaires transfrontalières par l'établissement de règles minimales communes relatives à l'aide judiciaire et à d'autres aspects financiers des procédures civiles. ${ }^{57}$ La Commission a également présenté le 3 mai 2002

56 Le champ d'application du règlement serait le même que celui de la Convention de Bruxelles de 1968 (devenue règlement CE du 20 décembre 2000), mais se limiterait aux créances non contestées (définition de cette notion dans l'Art. 3,4 $4^{\circ}$ ). Le principe est qu'une décision relative à une créance incontestée qui a été certifiée en tant que titre exécutoire européen dans l'Etat membre d'origine, est reconnue et exécutée dans les autres Exars membres sans qu'une procédure spéciale soit requise dans l'Etat membre d'exécution (Art. 40); pour être certifiée, la décision doit avoir force de chose jugée dans l'Etar d'origine, ne pas être incompatible avec certaines sections du règlement Bruxelles I en matière de compétence judiciaire, elle doit avoir été rendue à la suite d'une procédure qui satisfaisait aux conditions procédurales minimales posées dans la proposition de règlement ; enfin, la signification ou la notification des actes nécessaires doit avoir été effectuée en respect de l'Art. $31^{\circ}$ de la proposition si elle devait avoir lieu dans un Etat membre autre que celui d'origine. Il convient de relever que c'est donc à la juridiction de l'Etat membre d'origine de bien vérifier, avant de délivrer un titre exécutoire européen, que les normes minimales de procédure telles qu'énoncées dans le chapitre III de la proposition de règlement, ont bien été respectées (ces normes sont relatives aux modes de signification ou de notification de l'acte introductif d'instance, v. Art. $11^{\circ}$ et $12^{\circ}$, à la preuve de la signification ou de la notification, Art. $13^{\circ}$, d'une signification en temps utile pour que le défendeur puisse préparer sa défense, Art. $15^{\circ}$, d'une information en bonne et due forme du débiteur sur la créance, Art. $16^{\circ}$, sur les formalités procédurales nécessaires pour contester la créance, Art. $17^{\circ}$, ainsi que sur les formalités procédurales nécessaires pour éviter une décision par défaut de comparution à une audience, Art. 18 ${ }^{\circ}$; il existe également des normes minimales en matière de levée de la forclusion résultant de l'expiration de délais). Le chapitre IV de la proposition de règlement détaille la procédure d'exécution et l'accès possible à la justice au cours de cette procédure, qui est également apte à s'appliquer aux transactions judiciaires et aux actes authentiques.

57 V. COM (2002) 13 final, disponible sur le site internet europa.eu.int. L'idée est de permettre au plaideur transfrontalier d'obtenir plus facilement et plus rapidement l'aide judiciaire dans l'Etat du for s'il existe des coopérations efficaces entre les différentes administrations nationales concernées. Des actions de formation et d'information du grand public et des professionnels doivent également être envisagées. Le projet de directive veut assurer que le plaideur transfrontalier sera trairé de la même manière que s'il résidait dans l'Etat membre du for, et que les difficultés inhérentes au caractère transfrontalier du litige ne fassent pas obstacle à l'octroi de l'aide judiciaire. La directive ne concernerait que les litiges en matière civile et commerciale, quelle que soit la nature de la juridiction appelée à 
une proposition de règlement du Conseil relatif à la compétence, la reconnaissance et l'exécution des décisions en matière matrimoniale et en matière de responsabilité parentale, abrogeant le règlement $C E \mathrm{~N}^{\circ} 1347 /$ 2000 et modifiant le règlement $C E$ 44/2001 en ce qui concerne les questions alimentaires. ${ }^{58}$ Cette proposition tend à intégrer le règlement CE $N^{\circ} 1347 / 2000$, la proposition que la Commission avait formulée

statuer; elle s'appliquera aux personnes physiques, mais également aux personnes morales sans but lucratif établies sur le territoire d'un Etat membre lorsque l'action judiciaire vise la protection d'intérêts généraux juridiquement reconnus, et lorsqu'elles n'ont pas les ressources suffisantes pour faire face aux charges des procédures (Art. $15^{\circ}$ de la proposition). L'exposé des motifs énonce (p. 3) que « la proposition de directive est avant tout destinée à ga rantir un niveau approprié d'aide judiciaire dans les affaires transfrontalières. En vue de garantir ce niveau approprié, il est nécessaire d'assurer la compatibilité de certaines dispositions de droit national. Compte tenu de cette nécessité, la directive du Conseil est l'instrument législatifle mieux approprié ". Bien évidemment, les Etats membres peuvent prévoir des dispositions encore plus favorables pour les personnes candidates à l'aide judiciaire. L'exposé des motifs de la proposition fait en outre référence à l'Art. $47^{\circ} \$ 3$ de la Charte des droits fondamentaux de l'Union européenne qui prévoit qu'une aide judiciaire est accordée à ceux qui ne disposent pas de ressources suffisantes dès lors que cette aide est nécessaire pour assurer l'effectivité de l'accès à la justice (proposition p. 3 et 4). Les Etats membres peuvent établir des seuils de ressources au dessus-desquels le candidat à l'aide judiciaire est présumé pouvoir faire face aux charges du litige ; ces seuils doivent être établis en tenant compte de différents éléments objectifs tels que le coût de la vie et les coûts des procédures. Si ces conditions ne sont pas remplies, le candidat peut néanmoins apporter la preuve qu'il ne pourrait faire face aux charges du litige, notamment en raison des différences de coût de la vie entre les Etats membres de résidence et du for, auquel cas l'aide doit lui être accordée (Art. 130 de la proposition). L'aide judiciaire, selon le projet, doit comporter au minimum l'assistance effective d'un avocat et l'exonération ou la prise en charge des frais de procédure. Si l'aide judiciaire est accordée, elle doit couvrir toute la procédure, y compris les frais encourus pour qu'un jugement soit déclaré exécutoire ou soit exécuté. De même, le bénéficiaire de l'aide doit continuer à la percevoir si un appel est formé contre lui. Toute décision de rejet de la demande d'aide judiciaire doit être motivée (Art. $8^{\circ}$ de la proposition de directive). La proposition énonce qu'il « convient de ménager la possibilité pour les Etats membres de rejeter les demandes d'aide judiciaire concernant des actions manifestement non fondées, sans pour autant procéder à un préjugement de l'affaire en vue d'évaluer les chances de succès du candidat à l'aide judiciaire » (on sait en effet qu'un tel préjugement a été condamné par la Cour européenne des droits de l'homme dans l'affaire Aerts c/ Belgique du 20 juillet 1998. Comp. C. eur. dr. H., 19 septembre 2000, Gnaboré c/ France, D. 2001. 735, note F. Rolin). Les demandes d'aide judiciaire dans un Etat membre autre que celui dans lequel réside le justiciable sont énoncées dans un formulaire standard (Art. $11^{\circ}$ de la proposition).

58 COM (2002) 0110 (CNS), disponible sur le site internet europa.eu.int. 
sur la responsabilité parentale ${ }^{59}$ et l'initiative française sur le droit de visite qui avait été présentée en juillet $2000 .^{60}$ Le texte reprend telles quelles les dispositions sur le divorce contenues dans le règlement Bruxelles II ; elle intègre en, outre un système complet de règles sur la responsabilité parentale et l'initiative française sur le droit de visite. ${ }^{61}$ Le règlement Bruxelles II du 29 mai 2000 serait abrogé et remplacé par ce nouvel instrument, ceci afin de faciliter le travail des juges et des praticiens du droit. ${ }^{62}$ Un chapitre spécial serait réservé dans ce nouveau règlement aux cas d'enlèvement d'enfants, l'Art. $21^{\circ}$ prévoyant que dans une telle hypothèse, les juridictions de l'Etat membre dans lequel l'enfant avait sa résidence habituelle immédiatement avant son déplacement ou son non-retour conservent en principe ${ }^{63}$ leur compétence.

22. Il apparaît clairement que la Communauté européenne est extrêmement active en matière de mesures de coopération judiciaire civile. ${ }^{64}$ Peut-être même trop, ou en tout cas de façon trop hâtive, ${ }^{65}$ ce qui nuit à la qualité des normes émises. Il suffit de citer l'exemple du règlement Bruxelles I du 22 décembre 2000 dont la rédaction avait débuté avant que le Royaume-Uni et l'Irlande n'expriment leur volonté d'y être associés et qui, par mégarde, oublie de mentionner dans son article $69^{\circ}$ les conventions auxquelles ces deux Etats sont parties et qui sont remplacées

59 JO CE C 332 du 27 novembre 2001.

60 JO CE C 234 du 15 août 2000.

61 Il s'agit là de supprimer toute exigence d'exequatur en matière de droit de visite (ce qui correspond à l'initiative française de juillet 2000 en la matière, en vue de l'adoption d'un règlement du Conseil relatifà l'exécurion mutuelle des décisions concernant le droit de visite des enfants). Le droit de visite a été identifié par le Conseil européen de Tampere comme une des priorités en matière de coopération judiciaire, car il s'agit de répondre à un besoin social réel.

62 V. exposé des motifs p. 4.

$63 \mathrm{~V}$. les dérogations énoncées à l'Art. $21^{\circ}, \$ 2^{\circ}$ de la proposition de règlement. Selon l'Art. $23^{\circ}$, les juridictions de l'Etat membre où se trouve l'enfant enlevé statuent à bref délai sur une demande de mesure conservatoire introduite au titre de l'Art. $22^{\circ}, \$ 3$ (ce dernier texte dispose que le retour de l'enfant ne peut être refusé qu'en introduisant une demande de mesure conservatoire, dans un certain délai, devant les juridictions de l'Etat membre où se trouve l'enfant enlevé).

64 V. notamment le compte rendu par la Commission de ses activités en ce domaine sur le site internet europa.eu.int.

65 Comp. B. HEb, Aktuelle Perspektiven der europäischen Prozessrechtsangleichung, in : $J Z 2001$, p. 573 et spécialement p. 582 et s. 
par le règlement $\mathrm{CE}$ comme elles l'avaient déjà été préalablement par la Convention de Bruxelles de $1968 .{ }^{66}$ Les perspectives sont riches et prometteuses, mais on peut se demander si la Commission a suffisamment mûri les projets nombreux qu'elle entend faire adopter au plus vite; ${ }^{67}$ il n'est pas exclu que tant les citoyens de l'Union que les professionnels du droit soient rapidement dépassés par une production législative intense pas toujours suffisamment peaufinée. L'accès au droit, un des droits fondamentaux énoncés par la Charte européenne des droits fondamentaux, ne sera pas élargi par une forêt de normes confuses et complexes. Il suppose au contraire à la fois transparence et clarté.

23. L'accès au droit et la prévisibilité des compétences ou des procédures est également un des objectifs que poursuivent les modèles d'intégration mondiale. Celle-ci s'illustre à l'heure actuelle par deux grands projets ambitieux mais difficiles qu'il convient d'aborder, d'analyser et d'apprécier.

II. Procédure civile et modèles d'intégration mondiale recherchée : le projet de Convention mondiale sur la compétence, la reconnaissance et l'exécution, et le projet de Principes et Règles transnationaux de procédure civile ALI-UNIDROIT.

24. A l'heure actuelle, deux projets sont rédigés et débattus qui présentent une dimension mondiale d'intégration recherchée : il s'agit tout d'abord du projet de Convention de La Haye sur la compétence judiciaire, la reconnaissance et l'exécution des jugements en matière civile et commerciale, projet qui, nous le verrons, se heurte à un certain nombre de difficultés (1); ensuite est également élaboré un projet de Principes et Règles transnationaux de procédure civile, qui, débuté sous l'égide de l'American Law Institute, a bénéficié d'une extension de son champ de préparation par l'association d'UNIDROIT à son élaboration (2).

66 Sur cette étrangeté et ce " lapsus calami ", v. G. Droz et H. Gaudemet-Tallon, préc., Rev. crit. DIP 2001, p. 618, à qui les autorités compétentes de la Communauté ont expliqué qu'il s'agissait d'un oubli dû au fait qu'à un moment donné, comme le Danemark, le Royaume-Uni et l'Irlande s'étaient trouvés en position de "opting out ", c'est-à-dire de refus de participation au règlement. Le revirement britannique n'a pas été pris en compte pour modifier l'article $69^{\circ}$ du règlement énonçant les conventions entre Etats membres remplacées par le nouveau règlement!

${ }^{67}$ Dans le même sens, B. HEb, préc., JZ2001, p. 582. 


\section{Le projet de la Conférence de La Haye de droit international privé d'une Convention sur la compétence et la reconnaissance et l'exécution des jugements étrangers en matière civile et commerciale.}

25. C'est le 19 octobre 1996 que fut présenté à la dix-huitième session de la Conférence de La Haye de droit international privé la proposition des Etats représentés $\mathrm{d}$ «inscrire à l'ordre du jour de la dix-neuvième session la question de la compétence, de la reconnaissance et de l'exécution des jugements étrangers en matière civile et commerciale». ${ }^{68}$ Les travaux ont été débutés en juin 1997 par une Commission spéciale qui, après cinq sessions, adopta en octobre 1999 une version préliminaire de convention qui devait être examinée à l'automne 2000 lors de la dix-neuvième session de la Conférence de La Haye. ${ }^{69}$ Très vite, la délégation américaine a contesté la méthode de travail, la procédure traditionnelle de la Conférence et sa "forte tendance majoritaire" " ainsi que la nature de convention double ${ }^{71}$ du projet de 1999 qui n'avait aucune chance d'être acceptée par les EtatsUnis. Le projet serait "dans une impasse». ${ }^{72}$ Le développement rapide du commerce électronique et des nouvelles technologies poserait des difficultés

68 Acte final de la dix-huitième session, I, Travaux de la dix-huitième session, divers 29, 47 (La Haye, SDU Publishers, 1999).

69 Sur l'historique du projet et des remarques critiques sur la démarche initiale adoptée, v. A. Von Mehren, La rédaction d'une convention universellement acceptable sur la compétence judiciaire internationale et les effets des jugements étrangers : Le projet de la Conférence de La Haye peut-il aboutir ?, Rec. crit. DIP2001, p. 85. Sur le texte du projet initial de 1999 et le texte provisoire rédigé en 2001, v. le site internet de la Conférence de La Haye http:// www.hcch.net.

70 A. Von Mehren, préc., p. 87.

71 Les conventions doubles sont celles qui règlent à la fois la compétence directe et indirecte, et qui distinguent entre compétences obligatoires et compétences prohibées. $\mathrm{Au}$ contraire, dans la convention mixte, préconisée par la délégation américaine, les tribunaux d'un Etat signataire sont libres d'appliquer ou non tout fondement de compétence qui n'est ni obligatoire, ni prohibé (ce que l'on appelle la liste grise de compétences, la liste blanche étant celle des compétences obligatoires et la liste noire celle des compétences prohibées) ; il s'ensuivrait que le juge qui retient sa compétence sur le fondement d'une liste grise risquerait de voir sa décision non reconnue dans un autre Etat signataire de la Convention. Le modèle de convention mixte serait plus souple et plus facilement acceptable par un grand nombre d'Etats.

72 A. Von Mehren, préc., p. 89. 
économiques et juridiques qu'il convient de tenter de régler afin que la Convention projetée puisse réellement avoir une portée importante; les questions de propriété intellectuelle seraient encore ouvertes.

26. Le travail de la Commission spéciale de la Conférence de La Haye est parti d'un modèle, ${ }^{73}$ celui de la Convention de Bruxelles du 27 septembre 1968 adopté sous la forme d'une convention intergouvernementale entre les Etats membres de la Communauté économique européenne devenue depuis lors Communauté et Union européennes. Cette Convention, qui ne constitue pas du droit communautaire dérivé puisqu'elle a été adoptée par les Etats membres eux-mêmes et non pas les organes de la Communauté, est aujourd'hui présentée comme modèle de réussite en matière de compétence internationale et d'exequatur européen, même si elle présente quelques failles qui n'ont pas toutes été supprimées par le règlement du Conseil de l'Union européenne $\mathrm{N}^{\circ} 44 / 2001$ du 22 décembre 2000 se substituant à la Convention de Bruxelles.

27. Pour la délégation américaine chargée de négocier le projet de convention mondiale, l'union économique et politique qui permet aux Etats de l'Union européenne de s'entendre sur un nombre important de questions de compétence et de reconnaissance n'existe pas sur le plan mondial; au contraire, les systèmes juridiques des Etats participant aux négociations de La Haye sont extrêmement divers et s'opposent sur des questions fondamentales. ${ }^{74} \mathrm{Il}$ conviendrait d'opter pour une convention mixte permettant au juge saisi de retenir sa compétence en vertu de critères qui ne sont ni imposés, ni prohibés par les stipulations de la Convention. C'est dans cette voie que semble s'engager le projet aujourd'hui; mais il a pris beaucoup de retard et il est difficile de prévoir s'il sera concrétisé un jour par la rédaction d'une Convention soumise à la signature des Etats parties à la Conférence de La Haye. Du 22 au 24 avril 2002 s'est réunie la

73 Modèle «totalement inapproprié pour les travaux de la Commission spéciale" selon A. Von Mehren, préc., p. 92.

74 V. notamment A. Von Mehren, préc., p. 90, qui cite comme exemples la question de savoir si les règles de compétence doivent favoriser le demandeur ou le défendeur, ou encore celle de l'étendue du pouvoir discrétionnaire du juge pour exercer ou non sa compétence et en matière de reconnaissance des jugements étrangers. Cet auteur relève également (p. 91) que la conception américaine de la fonction sociale du pouvoir juridictionnel s'oppose fondamentalement à celle de la plupart des Etats membres de l'Union européenne. 
Commission I sur les Affaires générales et la politique de la dix-neuvième session de la Conférence de La Haye afin de décider de l'orientation future des travaux de la Conférence. ${ }^{75}$ Il a été décidé qu'un processus de travail informel serait mis en place en vue de préparer un texte qui serait soumis à une Commission spéciale au premier semestre 2003 ; ensuite se tiendrait une conférence diplomatique, si possible à la fin de l'année 2003. Aujourd'hui encore, au moins six domaines importants n'obtiennent pas de consensus au sein des négociations: la question de la compétence judiciaire dans le cadre d'internet et du commerce électronique; la compétence fondée sur l'activité; les contrats conclu entre les professionnels et les consommateurs, ainsi que les contrats de travail; les brevets, marques, droits d'auteur et autres droits de propriété intellectuelle; les rapports de la future Convention avec d'autres actes sur la compétence, la reconnaissance et l'exécution des jugements, et notamment les actes régionaux tels que les Conventions de Bruxelles et de Lugano ainsi que le nouveau règlement $C E$ du 22 décembre 2000 remplaçant la Convention de Bruxelles dans les rapports entre les Etats membres sauf le Danemark; enfin, la question de la bi latéralisation. ${ }^{76}$ La question se pose aujourd'hui de savoir si le champ de la Convention demeurera aussi large qu'initialement, ou s'il n'y aurait pas intérêt — si les négociations doivent aboutir- à réduire ledit champ, ce qui serait certes décevant, mais en même temps peut-être plus prometteur car plus réaliste. ${ }^{77}$

75 La situation est encore compliquée par le fait qu'en 2001, dix nouveaux Etats sont devenus membres de la Conférence de La Haye de droit international privé : Pérou, Brésil, Bélarus, Géorgie, Yougoslavie, Jordanie, Bosnie-Herzégovine, Sri Lanka, Lituanie et Fédération de Russie. Pour des détails sur l'avancement des travaux, v. Quelques réflexions sur l'état actuel des négociations du projet sur les jugements dans le contexte du programme de travail futur de la Conférence, févr. 2002, sur le site internet de la Conférence de La Haye www.hcch.net.

76 Jusqu'à présent, la démarche des négociateurs a consisté à réserver les questions vraiment problématiques pour la fin des négociations en espérant qu'elles seraient résolues une fois que le cœur de la Convention serait solidement bâti, mais la question est désormais posée de savoir si ces matières ne sont pas si fondamentales que leur étude devrait être faite dans le processus même de construction de la Convention, v. en ce sens Quelques réflexions sur l'état actuel des négociations du projet sur les jugements dans le contexte du programme de travail futur de la Conférence, févr. 2002, sur le site internet de la Conférence de La Haye www.hcch.net, document p. 6, $\mathrm{N}^{\circ} 8$.

$77 \mathrm{~V}$. notamment la note établie par le bureau permanent de la Conférence de La Haye de droit international privé Limpact d'internet sur le projet sur les jugements : nouvelles 
28 L'idée est pourtant séduisante d'unifier autant que faire se peut les règles de compétence et les conditions ainsi que la procédure de reconnaissance et d'exequatur d'un jugement étranger. ${ }^{78}$ Dans le texte provisoire établi par le bureau permanent de la Conférence de La Haye et les rapporteurs à la suite de la Conférence diplomatique de juin 2001, le projet présente 42 articles, dont certains avec plusieurs options lorsqu'aucun consensus n'a pu être atteint sur une version unique. La suppression de certains articles est également envisagée, voire déjà convenue entre les négociateurs. ${ }^{79} \mathrm{La}$ Convention aurait ainsi un premier chapitre définissant son champ d'application (matériel, Art. $1^{\circ}$, territorial, Art. $2^{\circ}$ ); un deuxième chapitre consacré à la compétence (Art. 30: for du défendeur ; Art. $4^{\circ}$ : élection de for, ${ }^{80}$ Art. $5^{\circ}$ : droit du défendeur à contester la compétence ; Art. $6^{\circ}$ : contrats ; Art. $7^{\circ}$ : contrats conclus par les consommateurs article qui pose des difficultés et dont plusieurs versions sont proposés-

pistes de réflexion, sur le site internet de la Conférence www.hcch.net. Ce texte indique qu'il " est essentiel que la Convention ne soit pas un frein à la poursuite de la croissance du secteur du commerce électronique, mais serve plutôt à nourrir son développement ". Or des experts et groupes internationaux ont exprimé leur crainte que la Convention, dans sa rédaction actuelle, ne soit préjudiciable plutôt que bénéfique à la croissance de ce secteur. La question cruciale en matière de compétence est de trouver le juste équilibre en ce qui concerne les juridictions compétentes pour les utilisateurs d'internet et pour les entreprises; dans la démarche de " pays d'origine ", la compétence ne peut être exercée que par les juridictions du pays où est située la source de la transmission ; dans la logique du " pays de destination " au contraire, la compétence peut être exercée partout où les informations et/ou biens et services sont reçus. Le projet d'octobre 1999 de Convention mondiale sur la compétence judiciaire et la reconnaissance ainsi que l'exécution des jugements adoptait une démarche de "pays de destination " à l'égard des contrats entre professionnels (Art. $6^{\circ} \mathrm{du}$ projet initial). Le texte provisoire de 2001 a atténué cette option en incluant une formule selon laquelle un défendeur n'est pas soumis à la compétence en liste blanche s'il prend des " mesures raisonnables " pour éviter cette compétence, mais le consensus n'a pas encore été établi sur cette proposition.

78 Pour un commentaire du projet, v. F-R. Grabau et J. Hennecka, Entwicklung des weltweiten Zuständigkeits- und Anerkennungsübereinkommens - Aktueller Überblick, RIW2001, p. 569.

79 Il en va par exemple ainsi de l'article 14 relatif à la pluralité de défendeurs et de l'article $16^{\circ}$ sur l'appel en garantie et l'intervention.

80 Sur cette question difficile et très importante en pratique, voir le document rédigé par le bureau permanent de la Conférence de La Haye Les conventions d'élection de for dans le contentieux international : leur utilisation et les problèmes juridiques qu'elles soulèvent dans le cadre du texte provisoire, sur le site internet de la Conférence www.hcch.net. 
; Art. $8^{\circ}$ : contrats individuels de travail ; Art. $9^{\circ}$ : succursales et activité commerciale habituelle; Art. $10^{\circ}$ : délits ; Art. $11^{\circ}$ : trusts ; Art. $12^{\circ}$ : compétences exclusives ; Art. $13^{\circ}$ : compétence pour ordonner des mesures provisoires ou conservatoires ${ }^{81}$ Art. $14^{\circ}$ : pluralité de défendeurs; Art. $15^{\circ}$ : demande reconventionnelle; Art. 16 ${ }^{\circ}$ : appel en garantie et intervention; Art. $17^{\circ}$ : compétence fondée sur le droit national ; Art. $18^{\circ}$ : compétences interdites ; Art. 19\%: autorité du tribunal saisi ; Art. $20^{\circ}$ : sursis à statuer tant qu'il n'est pas établi que l'acte introductif d'instance a été notifié au défendeur en temps utile et de telle manière qu'il puisse se défendre; Art. $21^{\circ}$ : litispendance; Art. $22^{\circ}$ : circonstances exceptionnelles pour refuser d'exercer la compétence); un troisième chapitre aborde la reconnaissance et l'exécution (Art. 23ㅇ définition du terme jugement; Art. $23 \mathrm{~A}$ : reconnaissance et exécution de mesures provisoires ou conservatoires ; Art. $24^{\circ}$ : jugements exclus du chapitre III ; Art. $25^{\circ}$ : jugements reconnus ou exécutés; Art. $26^{\circ}$ : jugements qui ne peuvent être reconnus ou exécutés; Art. $27^{\circ}$ : vérification de la compétence; Art. 27 A : comparution sans contestation de la compétence; Art. 28 : motifs de non reconnaissance ou d'exécution; Art. $29^{\circ}$ : pièces à produire ; Art. $30^{\circ}$ : procédure; Art. $31^{\circ}$ : frais de procédure ; Art. $32^{\circ}$ : aide judiciaire; Art. $33^{\circ}$ : dommages et intérêts ; Art. $34^{\circ}$ : divisibilité, c'est-à-dire reconnaissance ou exécution partielle; Art. $35^{\circ}$ : actes authentiques ; Art. 36०: transactions) ; enfin, un dernier chapitre comporte des dispositions générales telles que les rapports avec d'autres conventions (Art. 37०), l'interprétation uniforme (Art. $38^{\circ}$ à $40^{\circ}$ ), la clause fédérale (Art. $41^{\circ}$ ) et enfin la ratification et l'adhésion (Art. 42\%).

29. A cette démarche d'intégration mondiale s'opposent donc des difficultés notamment liées à la présence d'un bloc uni au sein des négociations, celui des Etats de l'Union européenne, dont le "vote groupé» a parfois été fustigé par certains. ${ }^{82}$ Mais la situation des pays de l'Union européennes au sein des négociations de La Haye est quelque peu

81 Le maintien de cet article est douteux ; il est possible que le projet de Convention ne traite aucunement des mesures provisoires ou conservatoires.

$82 \mathrm{~A}$. Von Mehren, préc., p. 99, qui relève "une tendance croissante au vote groupé» de la part des Etats membres de l'Union européenne, circonstance qui, selon lui, permet de comprendre en grande partie l'effer important que la Convention de Bruxelles a eu sur le projet de convention mondiale d'octobre 1999 ; l'auteur ajoute que, selon lui, «à long terme, le vote groupé est contre-productif, si l'on souhaite une convention accueillie par tous's. 
compliquée. En effet, au regard des nouveaux textes issus des réformes les plus récentes du Traité CE et du Traité d'Union européenne, il semble que désormais, dans les matières relevant de la coopération judiciaire dans les matières civiles, lorsque cela est nécessaire au bon fonctionnement du marché, les institutions de l'Union européenne soient compétentes pour établir des textes communautaires (Art. 650 Traité $\mathrm{CE}$ ), ${ }^{83}$ mais également

83 Cet article $65^{\circ}$ énonce que "les mesures relevant du domaine de la coopération judiciaire dans les matières civiles ayant une incidence transfrontière, qui doivent être prises conformément à l'article $67^{\circ}$ et dans la mesure nécessaire au bon fonctionnement du marché intérieur, visent entre autres à :

a) améliorer et simplifier :

- le système de signification et de notification transfrontière des actes judiciaires et extrajudiciaires;

- La coopération en matière d'obtention de preuves ;

- La reconnaissance et l'exécution des décisions en matière civile et commerciales, y compris les décisions extrajudiciaires;

b) favoriser la compatibilité des règles applicables dans les Etats membres en matière de conflits de lois et de compétence

c) éliminer les obstacles au bon déroulement des procédures civiles, au besoin en favorisant la compatibilité des règles de procédure civile applicables dans les Etats membres».

Sur la difficile question de la délimitation des compétences de la Communauté sur le fondement de l'article $65^{\circ}$ et sur celui de l'article $95^{\circ}$ Traité CE, v. notamment H.-P. Mansel, "Zum Systemwechsel im europäischen Kollisionsrecht nach Amsterdam und Nizza", in Systemwechsel im europäischen Kollisionsrecht, Munich, Beck éd., 2002, p. 3 et s. L'auteur relève à juste titre qu'il manque aux mesures fondées sur l'article $65^{\circ}$ la "légitimité parlementaire "; sur le fondement de l'article $95^{\circ}$, le Parlement européen se voit reconnaitre un véritable pouvoir de codécision, alors que la procédure d'adoption contenue dansl'article $67^{\circ}$ est beaucoup plus sommaire, même si elle suppose jusqu'au $1^{\text {er }}$ mai 2004 un (simple) droit pour le Parlement de s'exprimer sur le projet ainsi qu'un vote unanime au sein du Conseil. L'article $95^{\circ}$ fonde la compétence législative de la Communauté sur l'établissement et le bon fonctionnement du marché intérieur ; l'article $65^{\circ}$ fait, lui, également référence au "bon fonctionnement du marché intérieur ", ce qui crée des difficultés pour délimiter les deux fondements de compétence législative. Une des différences essentielles entre les deux articles est que les mesures fondées sur l'article $65^{\circ}$ ne concernent pas le Danemark, et ne concernent le Royaume-Uni et l'Irlande que s'ils manifestent expressément leur voilonté d'y participer. V. aussi sur la question J. Basedow, «Die Vergemeinschaftung des Kollisionsrechts nach dem Vertrag von Amsterdam", in : Systemwechsel im europäischen Kollisionsrecht, op. cit., p. 19 et s. Ce dernier insiste (p. 67) à juste titre sur les dangers d'étendre de façon excessive le champ d'application de l'article $65^{\circ}$ Traité $\mathrm{CE}$, en raison du fait que l'article $68^{\circ}$ applicable aux mesures prises sur le fondement de l'article $65^{\circ}$ limite dangereusement le droit de recours préjudiciel des juridictions nationales devant la Cour de Justice des Communautés européennes, ce qui constitue un risque pour l'unité de la jurisprudence. 
pour négocier des traités internationaux qui engageraient les Etats membres dès lors que de tels traités pourraient avoir des incidences sur le droit communautaire et son application. ${ }^{84}$ Le Titre IV du Traité CE, intitulé Visas, asile, immigration et autres politiques liées à la libre circulation des personnes, contient dans son article $61^{\circ}$ la volonté de créer progressivement un «espace de liberté, de sécurité et de justice». ${ }^{85}$ Ce domaine a été communautarisé; la coopération judiciaire dans les matières civiles est passée du troisième au premier pilier, ce qui ouvre de nouvelles perspectives législatives européennes permettant à des règlements communautaires de se substituer à des conventions internationales préexistantes. C'est ce qui s'est produit récemment avec l'adoption des règlements $\mathrm{N}^{\circ} 1347 / 2000$ du 29 mai $2000^{86}$ dénommé Bruxelles II et 44/2001 du 22 décembre $2000^{87}$ dit Bruxelles I. En règle générale, la compétence communautaire fondée sur le Traité est exclusive et irréversible, sauf à amender le Traité, si bien que les Etats membres ne sont pas habilités à adopter des mesures dans ces domaines sans une autorisation spécifique de la Communauté. La Cour de Justice des Communautés européennes avait d'ailleurs jugé, dans sa décision Commission c/ Conseil , ERTA, affaire 22/70 ${ }^{88}$ du 31 mars 1971, que chaque fois que la Communauté, en vue de réaliser une politique commune envisagée par le Traité, adoptait des dispositions édictant des règles communes, les Etats membres n'étaient plus habilités, en agissant individuellement ou même collectivement, à souscrire des obligations avec des Etats tiers. ${ }^{89}$ Certes, en matière de coopération

84 Sur cette question de répartition des compétences entre Etats membres de l'Union européenne et institutions de l'Union en matière de traités internationaux, v. notamment Ch. T. Kotuby, "External Competences of the European Community in the Hague Conference on Private International Law : Community Harmonization and Worlwide Unification ", NILR (Netherlands International Law Review) 2001, p. 1.

$85 \mathrm{Ce}$ Titre IV issu du Traité d'Amsterdam est entré en vigueur le $1^{\text {er }}$ mai 1999 , comme l'ensemble du Traité.

86 JOCE L 160 du 30 juin 2000, p. 19.

87 JOCE L 12 du 16 janvier 2001, p. 1.

88 Affaire 22/70, Rec. p. $263, \$ 28$.

$89 \mathrm{Il} \mathrm{semble} \mathrm{toutefois} \mathrm{que} \mathrm{cette} \mathrm{jurisprudence} \mathrm{ait} \mathrm{été} \mathrm{atténuée} \mathrm{par} \mathrm{la} \mathrm{Cour} \mathrm{dans} \mathrm{des} \mathrm{avis}$ $1 / 94$ (du 15 novembre 1994, Rec. 1994. I. p. 5267) et 2/92 du 23 mars 1995 (Rec. 1995. I. p. 525) : là, la Cour applique plus restrictivement la théorie de la compétence externe implicite et- insiste sur le fait que, en l'absence d'harmonisation complète d'un domaine, la Communauté n'acquiert une compétence externe exclusive que dans la mesure dans laquelle les normes communes ont été adoptées sur le plan interne (communautaire). 
judiciaire dans les matières civiles, l'article $65^{\circ}$ exige que les mesures adoptées soient nécessaires au bon fonctionnement du marché ; mais cela sera souvent le cas. En exerçant ses nouvelles compétences, la Communauté est également impliquée comme un ensemble à l'égard des Etats tiers, et le Traité permet à la Communauté d'adopter unilatéralement des règles de conflit afin de simplifier les cas de conflit au sein de la Communauté autant que pour protéger le droit matériel de l'Union européenne face au droit des Etats tiers. ${ }^{90}$ Il semble donc bien que la compétence externe de la Communauté existerait dans les matières couvertes par l'article $65^{\circ} \mathrm{du}$ Traité, ce qui signifierait qu'elle serait habilitée à négocier des conventions internationales dans ces domaines en lieu et place des Etats membres, ${ }^{91}$ même si l'on peut alors se demander quel rôle peut bien jouer l'article $293^{\circ}$ Traité CE qui, relevant du troisième pilier, énonce que «les Etats membres engageront entre eux, en tant que de besoin, des négociations en vue d'assurer, en faveur de leurs ressortissants:

$-[\ldots]$.

- la simplification des formalités auxquelles sont subordonnées la reconnaissance et l'exécution réciproque des décisions judiciaires ainsi que des sentences arbitrales ${ }^{9}{ }^{92}$

90 En ce sens, Ch. T. Kotuby, préc., p. 15. V. aussi les développements de cet auteur p. 16 et 17 .

91 Un certain nombre d'auteurs (v. notamment J. Basedow, "The Communitarization of the Conflict of Laws under the Treaty of Amsterdam," CMLR 37 (2000), p. 687 et spécialement p. 697 et s. ; B.HEb, Aktuelle Perspektiven der europäischen Prozessrechtsangleichung, $J Z 2001$, p. 573 et 574 . Comp. B. HEb, Die Europäisierung des internationalen Zivilprozessrechts durch den Amsterdamer Vertrag - Chancen und Gefahren $N J W 2000$, p. 23 et spéc. p. 29) ) considèrent que la compétence législative de la Communauté en matière de droit international privé pourrait également être fondée sur l'article 95 Traité CE (à condition que les mesures prises ne concernent pas exclusivement la libre circulation des personnes, v. art. $95,2^{\circ}$ ), qui impliquerait une autre procédure que celle de l'Art. $68^{\circ}$. La procédure de l'article $95^{\circ}$ permet en outre d'y inclure le RoyaumeUni, l'Irlande et le Danemark, Etats qui ont refusé de participer d'office aux mesures prises sur le fondement de l'article $65^{\circ}$ Traité CE. En pratique, l'Union européenne opte pour l'article $65^{\circ}$ ou pour l'article $95^{\circ}$ comme fondement d'une mesure de la façon suivante : les mesures détaillées qui concernent essentiellement le droit processuel international au sein de l'Union européenne (notion de procès dans me marché intérieur, Binnenmarktprozess) sont fondées sur la norme plus spéciale de l'article $65^{\circ}$. Les actes juridiques qui établissent seulement une harmonisation minimale au sein du marché intérieur, sans contenir de règles détaillées sur l'organisation de la procédure, sont adoptés sur le fondement de l'article $95^{\circ}$ (v. B. HEb, préc., JZ2001, p. 574). 
Le maintien de cet article paraît signifier que les Etats membres conservent une compétence de négociation de conventions internationales dans le domaine notamment de la reconnaissance et de l'exequatur, ce qui justifierait leur présence à la table des négociations de la Conférence de La Haye, sauf à retenir leur compétence seulement résiduelle pour les matières non civiles ni commerciales (administratives et pénales), ${ }^{93}$ la Commission européenne y assiste également, mais en qualité d'observateur. ${ }^{94} \mathrm{On}$ peut aussi estimer ${ }^{95}$ que, dans les domaines relevant de l'article $65^{\circ}$ du Traité $\mathrm{CE}$ (coopération judiciaire en matière civile), la compétence de négocier des conventions internationales appartient tant aux Etats membres qu'à la

92 Sur la porté résiduelle de l'article 293 Traité CE, v. J. BASEDOW, The Communitarization of the Conflict of Laws under the Treaty of Amsterdam, CMLR37 (2000), p. 687 et sépcialement p. 699 et s. L'auteur considère que le $\mathrm{n}^{\circ} 4$ de cet article, qui concerne la simplification des formalités en matière de reconnaissance et d'exequatur ne peut désormais s'appliquer qu'aux matières exclues du champ de la Convention de Bruxelles de 1968 et du règlement CE du 22 décembre 2000 (ainsi que de celui du 29 mai 2000 relatif à la rupture du lien matrimonial et à la responsabilité parentale), c'est-à-dire concrètement aux matières pénales, administratives et fiscales.

93 Selon l'analyse de J. Basedow, préc., ibid.

94 V. B. HEb, Die Integrationsfunktion des Europäischen Verfahresnrechts, IPRax 2001, p. 389 et plus spécialement p.396 : l'auteur relève que la Communauté européenne ne peut exercer sa nouvelle compétence externe au sein de la Conférence de La Haye, car elle n'en est pas membre et ne dispose que du statut d'observateur ; elle est donc " représentée " lors des négociations par les Etats membres, mais cette situation va sans doute être bientôt modifiée. V. aussi la déclaration commune du Conseil et de la Commisison quant aux négociations au sein de la Conférence de La Haye de droit international privé, qui établit un processus de coopération, au sein des négociations, qui « en aucun cas ne porte atteinte à l'étendue des compétences externes de la Communauté "(IPRax 2001, pp. 260 et s.).

95 Sur la question, v. J. Basedow, préc., p. 702 qui relève que la réponse à la question de savoir si la Communauté est habilitée à adopter des traités internationaux en matière de conflit de juridictions dans un cadre extérieur à l'Union européenne " is by no means clear ". L'auteur relève que la référence dans le Titre IV du Traité CE et dans son article $65^{\circ}$ (ainsi que dans l'Art. 95\%) au marché intérieur semble signifier que ces textes concernent avant tout les relations intra-communautaires. Néanmoins, l'objectif de ces articles semble impliquer une compétence des institutions communautaires pour adopter des règles de conflit bilatérales, et de négocier des conventions mondiales comme celle que projette la Conférence de La Haye de droit international privé. L'auteur en conclut que les institutions communautaires peuvent adopter des mesures étendant le règlement des conflits de lois au delà de l'Union européenne, en relation avec des Etats tiers, et a l'autorité nécessaire pour négocier avec des Etats tiers. 
Communautée ${ }^{96}$ (hypothèse de compétence concurrente ou partagée. ${ }^{97} \mathrm{Au}$ sein de la Conférence de La Haye, la présence de représentants des Etats membres est d'autant plus nécessaire que pour l'instant, la Commission européenne n'a que peu d'expérience en matière de droit international privé, ${ }^{98}$ ce qui rend donc la collaboration entre Etats membres et Commission à la fois souhaitable et utile.

30. Les intentions de la Communauté européenne paraissent claires. Le nouveau règlement $C E$ 44/2001 du 22 décembre 2000 en matière de compétence, de reconnaissance et d'exécution, dans son préambule, énonce que doit être assurée la continuité d'un système autonome et complet de compétence et de reconnaissance, et maintenues aussi limitées que possible les matières issues de son champ d'application. Ces déclarations montrent que la Communauté entend harmoniser complètement le domaine de la compétence juridictionnelle, de la reconnaissance et de l'exécution, ce qui —selon certains - ${ }^{99}$ laisse présumer l'existence d'une compétence externe exclusive de la Communauté, sur le fondement de ce nouveau règlement. Ce dernier prend d'ailleurs parti à propos des conventions en matière particulière auxquelles sont parties des Etats membres: $1^{\prime} A r t .71^{\circ} \mathrm{du}$ nouveau règlement dispose en effet que le règlement «n'affecte pas les conventions auxquelles les Etats membres sont parties et qui, dans des matières particulières, règlent la compétence judiciaire, la reconnaissance ou l'exécution des décisions"; ; ceci semble signifier, en comparaison avec l'article $57^{\circ}$ de la Convention de Bruxelles de 1968 qui énonçait que «la présente convention n'affecte pas les conventions auxquelles les Etats contractants sont ou seront parties [...]", que désormais, les Etats membres ne sont plus autorisés à adopter entre eux ou avec des Etats tiers des traités de compétence, de reconnaissance et d'exécution en des matières

96 En ce sens, et se fondant sur la nécessité d'une solution réaliste au problème de compétence, notamment dans le cadre de la Conférence de La Haye, v. H. Duitjer Tebbens, "Ein Ziviljustizraum in der Europäischen Union - auf Kosten einer Aushöhlung der internationalen Zusammenarbeit ?", in : Systemwechsel im europäischen Kollisionsrecht, Beck éd., Munich, 2002, p. 188.

97 "Concurrent or shared competence ", v. Ch. T. Kotuby, préc., p. 20. V. aussi J. Basedow, The Communitarization of the Conflict of Laws under the Treaty of Amsterdam, CMLR 37 (2000), p. 687 et spécialement p. 704.

98 En ce sens, Ch. T. Kotuby, préc., p. 29 .

99 Ch. T. Kotuby, préc., p. 23. 
particulières. ${ }^{100}$ Comme le relèvent certains auteurs, ${ }^{101}$ «les Etats membres de l'Union européenne ont perdu de leur souveraineté sur le plan diplomatique et ne peuvent plus librement négocier avec un Etat tiers un régime d'entraide réciproque en matière de conflit de juridictions».

31. La question se pose donc aujourd'hui de savoir si les Etats membres de l'Union européennes sont habilités à négocier au sein de la Conférence de La Haye de droit international privé, une convention mondiale sur la compétence juridictionnelle, la reconnaissance et l'exequatur des jugements, ${ }^{102}$ convention qui peut avoir des incidences sur l'application des règles communautaires (règlement CE Bruxelles I No 44/2001 du 22 décembre 2000) en la matière. En même temps, seuls des Etats peuvent — semble-t'il- être membres de la Conférence de La Haye; les statuts de cette dernière ne permettent pas encore la négociation et la conclusion de conventions par des organisations régionales telles que l'Union européenne; la Commission européenne ne pourrait donc pas pour l'instant participer aux négociations seule, ${ }^{103}$ sans la présence de représentants des Etats

100 Sur ces questions, v. F. Ferrand, in : Dalloz Action Procédure civile, Dalloz éd., Paris, 2002, $\mathrm{N}^{\circ}$ 1218. De même, alors que l'Art. 59\% de la Convention de Bruxelles de 1968 énonce que la Convention " ne fait pas obstacle à ce qu'un Etat contractant s'engage envers un Etat tiers, aux termes d'une convention sur la reconnaissance et l'exécution des jugements, à ne pas reconnaître une décision rendue, notamment dans un autre Etat contractant, contre un défendeur qui avait son domicile ou sa résidence habituelle sur le territoire de l'Etat tiers, lorsque, dans le cas prévu par l'article $4^{\circ}$, la décision n'a pu être fondée que sur une compétence visée à l'article $3^{\circ}$ deuxième alinéa ", l'article $72^{\circ} \mathrm{du}$ nouveau règlement $\mathrm{CE}$ du 22 décembre 2000 dispose, lui, que le règlement " n'affecte pas les accords par lesquels les Etats membres se sont engagés avant l'entrée en vigueur du présent règlement, en vertu de l'article $59^{\circ}$ de la Convention de Bruxelles, à ne pas reconnaître... ". Cette différence de formulation montre bien que désormais, les Etats membres ne peuvent plus négocier des accords isolés avec des Etats tiers.

101 G. Droz et H. Gaudemet-Tallon, «La transformation de la Convention de Bruxelles du 27 septembre 1968 en Règlement du Conseil concernant la compétence judiciaire, la reconnaissance et l'exécution des décisions en matière civile et commercialen, in : Rev. crit. DIP 2001, p. 601 et notamment p. 623.

102 Sur des doutes quant à la compétence de négociation des Etats membres, v. Ch. T. Kotuby, préc., p. 22 : "the ability of the Member States to enter into international agreements with third countries on the matters which are currently under negociation at the Hague Conference seems dubious".

103 Sur la nécessité pour les organisations internationales de s'adapter à l'évolution des compétences communautaires et par exemple, pour la Conférence de La Haye, d'accepter tant des clauses de déconnexion que la possibilité d'une adhésion de la Communauté 
membres plus expérimentés en matière de négociations internationales en droit international privé que la Commission européenne.

32. Les négociations au sein de la Conférence de La Haye ont également fat apparaître le problème de l'incidence de la Convention mondiale projetée sur le droit international privé communautaire. Le projet envisage une "disconnection clause» (clause de déconnexion) garantissant la priorité de la Communauté en toutes circonstances (ou en certaines circonstances selon les propositions, sur lesquelles il n'y a pas encore de consensus) et précise que les règles de la Convention ne peuvent affecter les mesures adoptées par la Communauté. Dans le projet de convention d'octobre 1999, plusieurs propositions de clause de déconnexion étaient formulées, par ex. en excluant l'effet de la Convention sur la réglementation communautaire: "the Convention does not affect the uniform laws based on special ties of a regional or other nature between the States concerned and to the instruments adopted by a community of States ; une seconde proposition énonçait que " a European instrument State shall apply the European instruments, and not the Convention, whenever the European instruments are applicable according to their terms » avec trois exceptions dans lesquelles même si le défendeur était domicilié dans un Etat membre de l'Union européenne, la Convention de La Haye devait quand même être appliquée. ${ }^{104}$

Dans le texte provisoire de 2001, quatre propositions sont formulées dans l'article $37^{\circ}$ réglant les rapports de la convention projetée avec d'autres conventions. ${ }^{105}$

européenne à la Conférence de La Haye de droit international privé, v. H. Duintjer Tebbens, préc., in : Systemwechsel im europäischen Kollisionsrecht, Beck éd., Munich, 2002, p. 192.

104 V. Art. $37^{\circ}$ du projet de 1999 : même si le défendeur est domicilié sur le territoire d'un Etar membre de l'Union européenne, a) les juridictions de cet Etat doivent appliquer l'Art. $4^{\circ}$ de la Convention mondiale même si la juridiction choisie par l'élection de for n'est pas une juridiction d'un Etat membre de l'Union européenne ; b) l'Art. $12^{\circ}$ de la Convention mondiale doit être appliqué même si la juridiction à compétence exclusive selon ce texte n'est pas une juridiction d'un Etat membre de l'Union européenne ; c) les Art. $21^{\circ}$ et $22^{\circ}$ de la Convention mondiale doivent être appliqués même si la juridiction en faveur de laquelle la procédure est suspendue ou la compétence est déclinée n'est pas la juridiction d'un Etat membre de l'Union européenne.

105 Proposition $1:$ : 1. La Convention ne déroge pas aux instruments internationaux auxquels des Etats contractants sont, ou seront parties et qui contiennent des dispositions sur les matières réglées par la Convention, à moins qu'une déclaration contraire ne soit faite par les Etats liés par un tel instrument. 
33. L'avenir du projet de Convention mondiale sous les auspices de la Conférence de La Haye est incertain. On peut espérer que les Etats parviendront à s'entendre sur un certain nombre de règles de compétence qui faciliteront les transactions internationales et le règlement des litiges y

2. Toutefois, la Convention l'emporte sur de tels instruments dans la mesurc où ceuxci préconisent des fors non autorisés aux termes de l'article $18^{\circ}$ de la Convention.

3. Les paragraphes précédents s'appliquent également aux lois uniformes reposant sur l'existence entre les Etats concernés de liens spéciaux, notamment de nature régionale, ainsi qu'aux instruments adoptés au sein d'une communauté d'Etats ". En vertu de cette proposition, la Convention future de La Haye prévaudrait en l'absence de déclaration contraire effectuée par les Etats parties aux instruments européens.

Proposition 2 : « 1. a) Dans cet article, la Convention de Bruxelles, le Règlement sur la compétence judiciaire, la reconnaissance et l'exécution des décisions en matière civile et commerciale de l'Union européenne, et la Convention de Lugano seront désignés globalement comme " les instruments européens".

b) Un Etat partie à l'une ou l'autre de ces Conventions ou un Etat membre de l'Union européenne auquel le Règlement susmentionné s'applique seront globalement désignés comme « les Etats soumis aux instruments européens ».

2. Sous réserve des alinéas suivants, un Etat soumis aux instrument européens doit appliquer ces instruments et non la Convention, dès lors que ces instruments sont applicables conformément à leurs dispositions.

3. Sauf lorsque sont applicables les dispositions des instruments européens sur

a) les compétences exclusives ;

b) la prorogation volontaire de compétence ;

c) la litispendance ou la connexité ;

d) les compétences protectrices des consommateurs ou des salariés ;

un Etat soumis aux instruments européens doit appliquer les articles $3^{\circ}, 5^{\circ}$ à $11^{\circ}, 14^{\circ}$ à $16^{\circ}$ et $18^{\circ}$ de la Convention lorsque le défendeur n'a pas de domicile dans un Etat soumis aux instruments européens.

4. Même si le défendeur est domicilié dans un Etat soumis aux instruments européens, un tribunal d'un tel Etat doit appliquer a) l'article $4^{\circ}$ de la Convention dès lors que le tribunal choisi ne relève pas d'un Etat soumis aux instruments européens ;

b) l'article $12^{\circ}$ de la Convention dès lors que le tribunal auquel l'article $12^{\circ}$ donne compétence exclusive ne relève pas d'un Etat soumis aux instruments européens ;

d) les articles $21^{\circ}$ et $22^{\circ}$ de la Convention dès lors que chaque fois que le tribunal au bénéfice duquel la procédure est suspendue ou la compétence déclinée ne relève pas d'un Etat soumis aux instruments européens ". En vertu de cette proposition, la règle de la Convention future de La Haye prévaudrait dans tous les cas.

Proposition 3 (disponible en anglais seulement) : "Judgments of courts of a Contracting State to this Convention based on jurisdiction granted under the terms of a different international convention (" other Convention") shall be recognized and enforced in courts of Contracting States to this Convention which are also Contracting States to the other Convention. This 
afférents. ${ }^{106}$ Parallèment, et non plus sur le plan du droit international privé des conflits de juridiction et de l'exequatur, mais en matière de règles de procédure dirigeant le déroulement du procès, un autre projet a vu le jour: celui mené conjointement par l'American Law Institute et UNIDROIT concernant des Principes et Règles transnationaux de procédure civile.

\section{Le projet ALI-UNIDROIT de Principes et Règles transnationaux de procédure civile. ${ }^{107}$}

\section{Historique - Lidée de proposer à l'adoption des Etats des règles} de procédure civile transnationale applicables aux litiges du commerce

provision shall not apply if, by reservation under Article [...], a Contracting State choosesa) not to be governed by this provision, or

b) not to be governed by this provision as to certain désignated other conventions".

Proposition 4: article 20: champ d'application territorial : “ 1 . Les dispositions du chapitre II s'appliquent devant les tribunaux d'un Etat contractant sauf si toutes les parties ont leur résidence habituelle dans cet Etat (ou sur le territoire d'une organisation régionale d'intégration économique qui est partie contractante en vertu de l'article [...]). Toutefois, même si toutes les parties ont leur résidence habituelle dans cet Etat contractant (cette Partie contractante" " : [...] ". Puis l'article 37\% A disposerait, en matière de rapports avec des conventions dans des matières particulières : « La présente Convention n’affecte pas les conventions auxquelles les Etats contractants sont ou seront parties et qui, dans des matières particulières, règlent la compétence judiciaire, la reconnaissance ou l'exécution des décisions ». ou encore «La présente Convention n'affecte pas l'application de toute autre Convention à laquelle les Etats contractants sont ou seront parties et qui, en relation avec certaines questions particulières, régissent la compétence ou la reconnaissance ou l'exécution des jugements, pourvu que l'application de cette autre Convention n'affecte pas les droits et obligations en découlant pour tout autre Etat partie qui n'est pas un Etat partie à cette autre Convention ". En vertu de cette proposition, la future Convention de La Haye ne prévaudrait que si une ou plusieurs parties ne sont pas habituellement résidentes dans l'Union européenne.

106 Comp. H. Schack, "Entscheidungszuständigkeiten in einem weltweiten Gerichtsstands- und Vollstreckungsübereinkommen" in : ZEuP 1998, p. 931, et spécialement p. 956, où l'auteur conseille aux Etats membres de ne ratifier l'éventuelle Convention mondiale qu'une fois que les Etats-Unis l'auront eux-mêmes fait ("Sonst steht zu befürchten, dal die Europäer einen von den Amerikanern geschmliedeten Klotz am Bein haben werden, den die Amerikaner selbst nicht akzeptieren".

107 Sur le projet, v. le dernier volume de la Revue de droit uniforme 2001 édité par UNIDROIT et contenant de nombreux articles relatifs au projet. V. aussi F. Ferrand, «Vers des règles transnationales de procédure civile ? Le projet de l'American Law Institute et 
international est née de la collaboration de deux collègues juristes: un Américain, le professeur Geoffrey Hazard, de la University of Pennsylvania Law School, et un Italien, le professeur Michele Taruffo, de l'Université de Pavie. Le point de départ de leur réflexion est que les coûts et soucis résultant d'un conflit juridique peuvent être limités grâce à la réduction des différences entre les systèmes juridiques. De tels rapprochements entre systèmes ont déjà largement été favorisés dans le domaine du droit matériel. Certes, il existe également des conventions internationales dans le domaine de la procédure civile, mais elles ont un domaine très limité, notamment celui de la compétence personnelle, et des conditions de reconnaissance et d'exécution d'un jugement étranger. Les auteurs du projet ont donc cherché à établir des règles que tous les pays intéressés pourraient adopter pour la solution des litiges relatifs aux échanges internationaux. Ils veulent offrir un "système équitable de procédure»" ${ }^{108}$ en proposant des règles qui «essaient d'exprimer, autant que possible, l'idéal d'un jugement impartial". ${ }^{109}$ Leur projet a été soumis à l'American Law Institute (ALI), qui a accepté de l'examiner et proposé à UNIDROIT ${ }^{110}$ un travail en commun en vue de l'adoption d'un texte final. Après avoir demandé au professeur Rolf Stürner de Fribourg-en Brisgau (RFA) une étude de faisabilité dont le résultat était plutôt encourageant, UNIDROIT a sollicité un certain nombre d'experts qui se sont réunis pour la première fois en mai 2000 à Rome afin de débattre du principe de règles transnationales de procédure civile et d'un éventuel choix à opérer entre règles détaillées et simples principes directeurs du procès transnational. De nouvelles sessions de travail se sont tenues en 2001 et en mai 2002.

35. Une volonté: la reconnaissance de principes fondamentaux de procédure - L'idée centrale du projet est celle d'équité et de "rassurance»

d'UNIDROIT» in : Droit et procédures, 2002, p. 4 ; R. Stürner, Modelregeln für den internationalen Zivilprozeb ? - Zum Stand eines gemeinsamen Vorhabens des American Law Institute und des Institut pour l'Unification du Droit privé (Unidroit), ZZP 112 (1999), p. 185. Sur les objectifs, les méthodes et les coûts de l'harmonisation, v. H. Kronke, Ziele-Methoden, Kosten - Nutzen : Perspektiven der Privatrechtsharmonisierung nach 75 Jahren UNIDROIT, in : JZ2001, p. 1149.

108 V. traduction française de l'introduction aux Principles and Rules of Transnational Civil Procedure, in : Vers un procès civil universel ? Les règles transnationales de procédure civile de l'American Law Institute, Paris, éd.. Panthéon-Assas, 2001, p. 215.

109 Ibid.

110 UNIDROIT réunit aujourd'hui cinquante huit Etats des cinq continents. 
du plaideur amené à agir ou se défendre devant une juridiction d'un Etat qu'il connaît peu et dont les pratiques procédurales pourraient être très éloignées de celles qu'il connaît dans son pays. ${ }^{111}$ Certains ont pu critiquer le projet en avançant que, finalement, il ne faisait que répéter le contenu de l'article $6,1^{\circ} \mathrm{CEDH}$ tel qu'interprété par la Cour européenne des Droits de l'Homme. ${ }^{112}$ Même si cela était, serait-ce un mal? En effet, les Principes et Règles transnationaux de procédure civile ont vocation à s'appliquer par exemple aux Etats-Unis, au Canada, à de nombreux pays africains, asiatiques, d'Amérique du Sud etc qui ne relèvent aucunement de la juridiction de Strasbourg. Nous serions alors dans un phénomène d'universalisation de la CEDH par imitation qui ne pourrait qu'être applaudi et rendre les Européens fiers d'avoir été les précurseurs en matière de droits fondamentaux de procédure.

36. Mais il ne semble pas que les Principes transnationaux de procédure civile se bornent à "recopier» le texte et la jurisprudence issue de l'article $6^{\circ} \mathrm{CEDH}$. Ils vont plus loin et garantissent un certain nombre de droits sur lesquels la Cour européenne de Strasbourg n'a encore jamais eu à statuer ou bien qu'elle a refusé de consacrer, par exemple en matière de "droit à la preuve». Conformément à la méthode américaine qui a largement été suivie dans leur formulation - ce qui a pu donner lieu à critique de la part de juristes européens - les différents principes fondamentaux retenus sont explicités, détaillés. Ils constituent une base substantielle de nature à encadrer de façon efficace le déroulement du procès civil transnational et à en garantir l'équité.

37. Les auteurs initiaux du projet, G. Hazard et M. Taruffo, avaient rédigé uniquement des règles, pour la plupart très détaillées, qui avaient donné lieu à nombre de critiques notamment au sein du groupe de travail réunissant les experts de l'American Law Institute et d'UNIDROIT. Suite à diverses suggestions, un nouvel avant-projet inclut des Principes fondamentaux de procédure qui précèdent les Règles plus détaillées. Les experts ont en effet considéré qu'avant d'édicter des règles précises, il

111 Comp. H. Kronke, préc., JZ2001, p. 1153 : « Es geht hier um die Schliessung von Gerechtigkeits- und Effizienslücken, welche sich in allen nationalen Zivilprozessrechten daraus ergeben, dass diese auf der immer häufiger unzutreffenden Annabme basieren, beide Parteien seien im Forumstaat ansässig ".

112 Comp. Vers un procès civil universel ? Les règles transnationales de procédure civile, op. cit., pp. 35 et s. 
convenait de tester une liste de principes et de voir si tous pouvaient être considérés comme fondamentaux pour le procès civil et comme soustendant les règles plus techniques qu'il faudrait ensuite étudier. C'est ainsi qu'ont été édictés vingt-quatre principes (entre-temps devenus vingt-huit !) qui font face à trente-six règles extrêmement détaillées. La question s'est alors posée des rapports entre ces deux corps de normes : les Principes constitueraient-ils une introduction aux Règles, ou bien seraient-il autosuffisants, et les Règles ne serviraient-elles alors que d'illustrations aux principes précédemment définis ? Lors de session de mai 2002, il a été décidé que les Principes seraient proposés à l'adoption par les Etats et que les Règles constitueraient une application facultative, une illustration des Principes que chaque Etat déciderait de retenir ou non, ou dont chaque Etat pourrait s'inspirer partiellement ou totalement pour aménager ses règles de procédure civile transnationale. Une telle solution de compromis satisfera les deux «courants»: les pays anglo-saxons qui préferent des règles détaillées à l'énoncé de principes purement théoriques, et les pays d'Europe continentale qui attachent une grande valeur à la formulation de principes et sont réticents envers des règles trop poussées dans le détail. ${ }^{113}$ Des règles détaillées pourront en outre s'avérer précieuses pour les Etats du monde non encore versés dans l'utilisation de droits fondamentaux de procédure, et qui pourraient avoir des difficultés à concevoir seuls la mise en application concrète des principes énoncés.

38. L'essentiel du procès équitable est contenu dans les principes transnationaux de procédure civile, qui au cours de leur préparation se sont transformés, d'une liste d'une ou deux pages, en formulation très détaillée qui pourrait s'auto-suffire. Les principes constituent ainsi un dénominateur commun fédérateur assez poussé. Le projet énonce aujourd'hui vingt-huit principes. Le premier détermine le champ d'application desdits principes (litiges nés d'opérations commerciales

113 V. l'introduction des Principes et Règles parue sur le site de l'American Law Institute (http : //www.ali.org), p. 15 du document, X. UNIDROIT Partnership ("The Principles generally apply to the civil-law mentality. Common-law lawyers may be less familiar with this sort of generalization and abstraction. But since the Principles and Rules are here being developped simultaneously, the relation between generality and specification is more sharply illuminated. The Principles are interpretive guides to the Rules and could be adopted as principles of interpretation. They could also be adopted as guidelines in interpreting existing national codes of procedure. Correlatively, the Rules can be considered as an exemplification of the Principles "). 
transnationales).Les autres principes tracent les contours du procès équitable en posant des exigences envers le tribunal et la procédure suivie devant lui, mais aussi envers les parties ou à leur profit.

39. Les principes fondamentaux retenus: tout d'abord, le principe $\mathrm{N}^{\circ}$ 2 affirme l'exigence de l'indépendance, l'impartialité et la compétence du tribunal $^{114}$ - De façon plus originale par rapport au droit français et européen, l'alinéa 2 impose que les juges saisis d'un litige transnational aient une expérience juridique substantielle et des connaissances juridiques adéquates. Cette exigence tend clairement à écarter le système américain du jury, si décrié en Europe. Elle risque toutefois aussi de conduire à la suppression de collèges de magistrats composés exclusivement de juges non professionnels. Le principe 3, lui, est consacré à la détermination de la compétence du tribunal envers les parties. Le principe 4 consacre l'égalité procédurale des parties, ${ }^{115}$ le principe 5 leur droit à l'assistance d'un conseil. Le principe 6 est consacré à la notification et au droit d'être entendu, ${ }^{116}$ tandis que le principe 7 , dans la droite ligne de l'article $6,1^{\circ} \mathrm{CEDH}$, impose la célérité de la justice. Les différentes phases du procès sont énoncées dans le principe $8 .{ }^{117}$ Le principe 9 définit le mode d'introduction de l'instance et la délimitation de l'objet du litige. ${ }^{118}$ Dans le principe 10

114 "Le tribunal doit bénéficier d'une indépendance lui permettant de résoudre le différend selon les faits et les moyens de droit allégués " (al. $1^{\mathrm{er}}$ )

115 ; «le tribunal doit être impartial " (al. 4 du principe). L'alinéa 4 garantit en outre une procédure permettant de mettre en cause la partialité des juges (par exemple récusation).

116 Le tribunal doit assurer à toutes les parties (quelle que soit leur position procédurale) les mêmes " aptitudes procédurales, en demande et en défense " en vertu des règles de procédure applicables, et sans considération de nationalité, de résidence ou de nature du litige. De même, la cautio judicatum solvi est expressément prohibée.

117 Le projet a voulu garantir l'information (Due notice) des parties quant au déroulement de la procédure : "Lors de l'introduction de l'instance, les parties doivent, par des moyens raisonnablement en mesure de garantir son efficacité, recevoir notification des demandes et défenses émanant de leur adversaire. Les parties reçoivent notification de tous les développements ultérieurs importants de l'instance". Le défendeur notamment doit être informé du risque d'un jugement par défaut. Ce principe $n^{\circ} 6$ énonce également la règle du contradictoire comme obligation des parties puisqu'il impose à chaque partie de faire connaitre à l'autre en temps utile tous les éléments de fait ou de droit sur lesquels elle fonde ses prétentions et défenses, et ce " afin que l'adversaire puisse organiser sa défense avant l'audience finale » (6.6). La possibilité des ordonnances sur requête (= non contradictoires) est toutefois expressément réservée (6.7).

118 Il s'agit de la phase introductive, de la phase intermédiaire et de la phase finale. 
est abordé l'office du juge dans la conduite de l'instance ${ }^{119}$ et le principe 11 tire les conséquences d'un défaut ou d'un refus d'une partie de participer à l'instance. ${ }^{120}$ Dans le même esprit, le principe 12 aborde la responsabilité des parties ${ }^{121}$ (se conduire loyalement dans leurs relations entre elles et envers le tribunal; chercher avec le tribunal une solution du litige efficace, équitable, et raisonnablement rapide; énoncer les moyens de fait, de droit et les preuves au soutien de leurs prétentions [...]). En matière probatoire, le principe 13 règle l'accès aux éléments d'information et à la preuve: est affirmé le droit du tribunal et de chaque partie à un «accès général aux

119 L'énoncé fondamental en matière d'introduction de l'instance est l'interdiction de saisine d'office du tribunal, qui ne peut voir introduire une instance devant lui que par la demande d'un plaideur. Il est intéressant de constater que ce principe, qui est plutôt innovant, rejoint la jurisprudence de la Cour des droits de l'homme de Strasbourg qui tend à considérer avec méfiance la saisine d'office par les tribunaux sous l'angle de la partialité que laissent souvent apparaître les termes et motifs de la citation à comparaître. On ne saurait que saluer cette disposition en matière de litige commercial. Lal. 2 du principe 9 ajoute - et ce en vertu du principe dispositif -, que l'objet du litige est déterminé par les demandes et défenses des parties, telles que présentées dans l'acte introductif d'instance et dans les conclusions en défense. Les demandes et défenses peuvent être modifiées dans un délai raisonnable en cas de motif sérieux. Toujours en vertu de la liberté des parties et de leur mâtrise sur le litige civil, l'art. 9.4 leur réserve la possibilité de mettre un terme au litige par retrait, admission de responsabilité totale ou partielle ou par accord amiable. Ce principe consacrant la maxime dispositive n'a rien de novateur mais méritait de figurer au rang des principes fondamentaux de procédure.

120 Dans la droite ligne des évolutions consacrées par le Nouveau Code de procédure civile français, les Principes prévoient que "le tribunal conduit activement l'instance; il exerce un pouvoir discrétionnaire afin de pouvoir mettre fin au litige loyalement et dans un délai raisonnable " (exigence de l'article $6,1^{\circ} \mathrm{CEDH}$ ). Un tel rôle actif du juge civil a également été renforcé tout récemment dans la réforme allemande de la procédure civile du 27 juillet 2001 (BGBl. 2001. I. p. 1887. V. notamment la modification du $\$ 139$ ZPO (Code allemand de procédure civile). On comprend en effet la nécessité d'un tribunal actif et quelque peu directif puisque le litige doit être tranché dans un délai raisonnable qui, pour être tenu, requerra parfois des injonctions ou initiatives autres du juge. Ce rôle actif du juge correspond aussi à la notion d'équité du procès dans la mesure où il est également exercé afin d'aboutir à la vérité.

121 Inspiré de l'idée anglo-saxonne de " contempt of Court ", ce principe permet au tribunal de sanctionner le plaideur qui s'abstient ou refuse de déférer à l'injonction du tribunal ou qui commet tout autre abus de procédure. L'alinéa 2 impose toutefois que les sanctions soient raisonnables (concept flou) et proportionnelles à l'importance et à la gravité de l'agissement reproché et de l'intention de son auteur. Divers exemples de « sanction appropriée "s sont cités dans l'Art. 11.3. 
informations pertinentes pour le litige et aux preuves non couvertes par une obligation de confidentialitém. Les parties peuvent elles-mêmes présenter toutes preuves pertinentes dans les limites du raisonnable, et même faire personnellement des dépositions à valeur probatoire. L'audition des parties, témoins et experts se déroule logiquement selon la loi du for (13.3), mais l'art. 13.3 garantit aux parties la faculté de poser des questions additionnelles, ce qui est étranger à notre tradition juridique et judiciaire continentale, mais est une formulation douce du principe de "cross examination» anglo-saxon. Le principe $\mathrm{N}^{\circ} 13$ est essentiel dans la mesure où il reconnaît en outre à une partie le droit de demander à l'autre partie ou à un tiers «la production de toute preuve directement pertinente, non couvertes par des règles de confidentialité, et qui se trouvent en la possession ou sous le contrôle d'une partie ou d'un tiers». La production d'un élément de preuve ne peut être écartée au motif qu'elle serait défavorable à la partie ou à la personne requise. Force est de constater que la Cour de Strasbourg ne s'est jamais engagée aussi loin dans la consécration d'un véritable droit à la preuve destiné à permettre une solution correcte du litige. Le principe 14 est consacré à la confidentialité et aux immunités en matière de production de preuve. ${ }^{122}$

40. Le principe 15 aborde la jonction d'instance et l'intervention, le principe 16 les dépositions écrites et orales des parties. Le principe de publicité de la procédure est également proclamé (principe 17). Le principe 18 aborde la charge de la preuve, qui en principe revient classiquement à la partie qui allègue les faits au soutien de sa prétention. Le principe 19 traite des devoirs respectifs des parties et du juge dans la détermination des éléments de droit et de fait. ${ }^{123}$ En principe 20 est abordé le jugement

122 Les parties se voient imposer un « devoir de loyauté " envers le tribunal et les autres parties ; elles doivent coopérer avec le juge dans la conduite de l'instance et dans l'administration de la preuve. Elles doivent notamment alléguer les faits et produire les preuves au soutien de leurs prétentions et défenses, notifier au tribunal et aux autres parties les preuves et moyens de droit qu' elles invoquent (respect de la contradiction). Le principe $N^{\circ} 12$ précise en outre que les parties livrent un exposé détaillé, en fait et en droit, dans l'acte introductif d'instance et les conclusions en défense. Est également admise la théorie du fait constant, dans une mesure beaucoup plus large que celle que consacre la jurisprudence française.

123 La question a été largement débattue lors des réunions d'experts à Rome, experts qui ont tous admis la nécessité de limiter dans certaines circonstances le droit à la preuve. Les cas les plus classiques, fondés tantôt sur le droit continental (secret professionnel, droit de ne pas s'auto-incriminer), tantôt sur le droit anglo-saxon (notamment sur la formulation 
— qui doit être rendu dans les plus brefs délais — ainsi que sa motivation en fait et en droit. La recherche de transaction et son encouragement par le tribunal sont abordés en principe 21 , dans l'esprit moderne ${ }^{124}$ très favorable aux modes alternatifs de résolution des litiges. Le principe 22 traite des frais et dépens et énonce que la partie gagnante a droit au remboursement d'une partie substantielle des frais réellement et raisonnablement engagés. Le principe 23 énonce que le jugement a un caractère définitif même s'il est susceptible de recours, et qu'un jugement définitif est immédiatement exécutoire. ${ }^{125}$ Le droit d'appel est soumis à la loi du for (principe 24). Le principe 25 aborde les questions de litispendance et de chose jugée; le principe 26 pose l'exigence d'une exécution efficace de la décision rendue. ${ }^{126}$ Le principe 27 prévoit la reconnaissance dans les autres Etats des jugements rendus dans un Etat signataire selon les mêmes modalités que les autres jugements et mesures provisoires du for, ce qui semble aller très loin et ne sera sans doute pas admis si facilement par les Etats. Enfin, le principe 29 prévoit une coopération judiciaire internationale. ${ }^{127}$

41. L'apport supplémentaire des Règles - Par rapport à des Principes extrêmement détaillés et dont peut parfois être contestée la nature de

de la confidentialité des communications entre le plaideur et son conseil, qui semble aller de soi en droit continental) ont été retenus.

124 Ce principe impose au tribunal de déterminer, après consultation des parties, le fondement juridique de la décision. Le tribunal est autorisé à fonder sa décision sur des moyens de droit, de fait ou de preuve non proposés par les parties, mais seulement si cellesci ont été mises en mesure de les commenter et, au besoin, de modifier leurs prétentions, moyens et défenses (illustration du principe contradictoire). Le juge se voit également (al. 3) reconnaître le pouvoir, comme dans de nombreux droits continentaux, d'ordonner l'administration de la preuve à la demande des parties ou d'office. On retrouve ainsi le rôle actif du juge, tel que formulé au principe 10.

$125 \mathrm{~V}$. par ex. la réforme allemande de la procédure civile du 27 juillet 2001, BGBl. 2001. I. 1887, et notamment $\$ 278$ nouv. ZPO (Gütliche Streitbeilegung, Güteverhandlung, Vergleich). Il eût sans doute été plus logique de placer ce principe immédiatement après l'article $10^{\circ}$ relatif à l'office du juge dans la conduite de l'instance.

126 Toutefois, le tribunal ou la juridiction d'appel, d'office ou à la demande de la partie contre laquelle la décision a été rendue, peut suspendre l'exécution pendant l'instance d'appel si cela s'avère nécessaire dans l'intérêt de la justice (22.4). En outre, le tribunal peut exiger le versement d'une caution appropriée, ou toute autre garantie, de l'appelant pour accorder une suspension de l'exécution forcée, ou de l'intimé pour refuser une telle suspension (23.5).

127 Le texte parle d'une exécution " rapide, proportionnelle et efficace des mesures provisoires, des condamnations pécuniaires, y compris autres frais, ou des ordonnances ". 
principe véritables, quelle peut être l'apport de Règles encore plus précises? Force est de constater que la méthode suivie (élaboration de règles initialement par les collègues Hazard et Taruffo, puis travail de rédaction de Principes fondamentaux essentiels et indispensables à un procès équitable, et enfin réexamen des règles à la lumière des principes formulés - cette dernière étape ayant débuté en mai 2002) a conduit à des répétitions, voire parfois à des contradictions entre les deux corps de normes. Pourtant, alors que les Principes ne contiennent aucune subdivision interne, les Règles, elles, sont plus structurées, et divisées en sept points: A. Standards d'interprétation; $\mathrm{B}$. Champ d'application des règles (ce qui peut paraître superflu dans la mesure où ce champ est déjà défini dans les principes); $C$. Compétence personnelle, jonction d'instance et intervention; $D$. Composition et pouvoirs du tribunal; E. Phase introductive; F. Audience plénière; G. Appel et procédures postérieures au jugement.

42. Les trois dernières parties reprennent donc les trois étapes du procès: E. phase introductive; F. Audience plénière (Trial); G. Appel et procédures postérieures au jugement. Elles sont utiles car elles détaillent la procédure et donnent des règles précises pour sa conduite; elles peuvent donc éclairer et inspirer les Etats qui sont peu familiers des principes fondamentaux et de l'idée d'équité du procès. S'il suffit en effet, pour les Etats assez avancés dans l'application des droits de l'homme et des libertés fondamentales en matière procédurale d'avoir une «liste» de principes à respecter, quitte à choisir la voie la plus opportune pour ces Etats dans la concrétisation du principe, nombre de pays manquent d'expérience dans la réalisation de l'équité processuelle et ont besoin de suggestions pratiques que contiennent les Règles.

Il en va ainsi des règles sur le début de l'instance, qui supposent la notification de la demande au défendeur en lui indiquant le délai pour répondre et la sanction possible d'un jugement par défaut (Art. 11\%). Il en va de même des détails quant au contenu de la demande (Art. 120) et des conclusions en défense ou demandes reconventionnelles (Art. 13\%: le texte suggère un délai de soixante jours pour le défendeur à compter de la notification de la demande pour répondre, avec possibilité d'extension dudit délai). L'hypothèse de modification de ses demandes ou de ses défenses par une partie est abordée dans le détail, avec proposition de délai pour la réponse de l'adversaire. Ce texte (Art. 140) sera sans doute revu afin de le mettre en complète adéquation avec les Principes, qui sont plus sévères quant à l'admission de telles modifications. La procédure par 
défaut n'est autorisée, qu'après des vérifications précises du tribunal et un relevé de forclusion est envisagé.

La phase préparatoire aborde aussi les propositions d'accord amiable en proposant toute une procédure à suivre, avec mention des moindres détails tels que les délais dans lesquels l'offre doit être considérée comme valable, ceux pour répondre, pour révéler au tribunal, une fois qu'il aura rendu le jugement, l'offre qui avait été faite et les sanctions ${ }^{128}$ susceptibles d'être prononcées par ledit tribunal dans l'hypothèse où le destinataire de l'offre n'aurait pas obtenu un jugement plus avantageux que l'offre amiable qui lui avait été soumise.

Les mesures provisoires sont également traitées de façon exhaustive ou presque, avec possibilité d'ordonnance rendue à l'issue d'une procédure non contradictoire dans un premier temps (Art. 17\%). Les Règles apportent des éclairages utiles sur différents points du procès : les décisions que le tribunal peut rendre dès la phase préparatoire (par exemple à propos de sa compétence, de l'applicabilité des Règles transnationales etc); la «disclosure» des éléments de preuve, et leur échange entre les parties (Art. $20^{\circ}$ ), la procédure à suivre pour la demande de production de certaines preuves, les dépositions et attestations écrites des témoins (Art. 22\%), caractère pertinent et admissibilité des preuves (Art. 240), expertise (Art.25\%), "Evidentiary Privileges» (confidentialité et immunités, Art. $23^{\circ}$ et $26^{\circ}$ ), administration et effets de la preuve (Art. 27\%), injonctions adressées aux tiers en matière de preuve, de mesure provisoire ou autre (Art. $28^{\circ}$ ).

43. La partie F. consacrée à l'audience plénière (Trial) est, elle, plus sommaire. Elle se compose de quatre articles seulement, qui traitent notamment de l'audience finale unique (Art. $2^{\circ}$ ), du procès verbal d'administration des preuves lors de l'audience finale; des règles concernent l'enregistrement de l'audience (Art. $30^{\circ}$ ). L'Art. $31^{\circ}$ aborde la discussion finale et le jugement. Enfin, l'Art. $32^{\circ}$ traite de la question du remboursement éventuel des frais, dépens et honoraires d'avocat.

128 " Les tribunaux d'un Etat qui a reconnu ces Principes doivent prêter leur assistance aux tribunaux de tout Etat étranger devant lesquels se déroule un procès conformément aux présents principes. Ceci comprend l'octroi de mesures provisoires et conservatoires, ainsi que la coopération à lidentification, la préservation ou à la production de preuves". On peut s'interroger sur les principes 27 et 28 , le premier semblant -de façon excessive-assimiler les jugements étrangers aux jugements du for (mais n'est-ce pas une maladresse de rédaction ?), le second posant une règle idyllique mais difficilement réalisable en pratique. La critique est également formulée quant à l'incorporation dans le projet de règles de compétence, de reconnaissance ou d'exécution à l'étranger. 
44. Enfin, les Règles abordent dans une dernière partie les phases ultérieures de la procédure, c'est-à-dire la question des voies de recours et de l'exécution du jugement. Contrairement aux Principes, la règle 33 semble autoriser de façon générale l'appel contre une décision de première instance ; en revanche, elle pose, comme les principes, la règle de limitation de l'effet dévolutif de l'appel aux demandes, défenses et demandes reconventionnelles présentées en première instance et écarte les nouvelles preuves sauf «to prevent manifest miscarriage of justice». Là encore, il conviendra d'harmoniser les deux corps de normes et d'opter pour une solution rédactionnelle précise. Une voie de recours après appel (further appellate review, pourvoi en cassation, Revision, cassazione etc), qui se limiterait à un contrôle en droit, existera seulement si la loi du for le prévoit. Les règles ne prennent donc nullement position sur ce point, ce qui paraît logique dans la mesure où les principes laissent déjà eux-même la recevabilité de l'appel dépendre de la lex fori.

Est également envisagée la nullité d'un jugement (nullification of judgment), mais seulement si le requérant a agi avec diligence et invoque une des causes de nullité limitativement énumérées à l'Art. $34^{\circ}$ des règles.

Le caractère immédiatement exécutoire du jugement de première instance est réaffirmé dans les Règles (Art. 38\%), mais une exception de sursis à exécution prononcé par la juridiction de première instance ou par la cour d'appel est prévue lorsqu'un tel sursis apparaît nécessaire «dans l'intérêt de la justice». ${ }^{129}$ Ceci est absolument similaire au contenu des Principes; les Règles ajoutent seulement une possibilité pour le tribunal d'intervenir en cas de défaut d'exécution de la part du perdant à l'issue d'un certain délai. ${ }^{130}$ Enfin, l'article $39^{\circ}$ reprend l'idée de coopération judiciaire internationale contenue dans l'article $28^{\circ}$ des principes, mais de façon plus concise. Un point est prévu dans les Règles et n'a pas été retenu dans l'article $28^{\circ}$ des Principes: la possibilité pour les tribunaux d'Etats n'ayant pas reconnu les règles transnationales d'exécuter des décisions facilitant la procédure pendante devant les tribunaux d'un autre Etat, car il s'agit là d'un vou pieux sans aucune utilité pratique: les Etats n'ayant pas adopté les Règles transnationales feront ce qu'ils décident au regard de

129 Les sanctions portent essentiellement sur le remboursement des frais engagés dans la procédure (Art. 16.6 des règles).

130 Le sursis à exécution peut être soumis à garantie ou consignation fournie par l'appelant (Art. 38.4). 
leurs règles de droit international privé et des éventuelles conventions biou multilatérales qu'ils ont pu ratifier.

45. Le projet est ambitieux. Il tend à créer dans le monde entier une assise commune du procès transnational en matière commerciale, assise garantissant un certain nombre de principes d'équité processuelle. En ce sens, le projet n'est pas de pure technique procédurale. Il contient, certes, plusieurs règles assez techniques telles que celles qui concernent les procédures de transaction, ou encore les frais et dépens. Mais le projet réaffirme aussi des valeurs fondamentales qui ont déjà été dégagées par la Cour européenne des droits de l'homme à partir de l'article 6, $1^{\circ} \mathrm{CEDH}$; il est parfois plus novateur, par exemple en matière de reconnaissance d'un droit à la preuve qui va dans le sens d'un procès civil tendu vers la recherche de la vérité. Les droits fondamentaux, cela nous le confirme, irriguent bien le champ processuel dans lequel ils ont toute leur place dans le sens d'un véritable accès équitable à la justice. Il est à espérer qu'il séduira les Etats et participera à une harmonisation de la procédure civile dans le sens de plus de transparence, plus de prévisibilité et une véritable égalité procédurale entre des parties qui auront bien souvent des nationalités et des résidences ou domiciles différents. Ce modèle d'intégration mondiale recherchée, tout comme celui projeté par la Conférence de La Haye de droit international privé, pose $t$-il davantage de problèmes que des textes d'intégration régionale? Quel modèle d'intégration choisir pour la procédure civile?

\section{Quels modèles d'intégration choisir?}

46. Comme le relève le Secrétaire général d'UNIDROIT, Herbert Kronke, ${ }^{131}$ nous connaissons très peu de choses sur les rapports entre harmonisation mondiale du droit privé et intégration régionale. Aucune

131 Le tribunal peut alors ordonner certaines mesures telles que l'obligation pour le débiteur de révéler la teneur de son patrimoine, ou encore une pénalité pécuniaire ; des tiers peuvent également se voir ordonner par le tribunal de délivrer des informations sur le patrimoine du débiteur. Toutefois, dans le même esprit que la jurisprudence de la Cour européenne des droits de l'homme (CEDH, 31 juillet 2001, Mortier c/ France, Droit et procédures 2002, p. 25, à propos de l'art. 1009-1 NCPC), les Règles (art. 38.2.4) prévoient qu'aucune pénalité ne peut être imposée à celui qui démontre au tribunal son impossibilité financière ou autre d'exécuter la décision rendue). 
étude systématique des liens entre les objectifs et degrés variables d'intégration économique d'une part et le mandat mondial de certaines organisations spécifiques n’a jusqu’à présent été réalisée. La question ne peut donc être abordée que de façon théorique et sans éléments chiffrés précis. Toutefois, un certain nombre de points peuvent être observés. Il est des arguments pragmatiques qui plaident en faveur d'une intégration régionale poussée (1) dont les effets pourront toutefois être à la fois positifs et pervers (2). Il conviendra de se demander si, dès lors qu'il existe des effets pervers à l'intégration d'abord régionale, les conséquences d'une recherche d'abord mondiale d'harmonisation sont meilleures (3).

\section{Les arguments pragmatiques en faveur d'une intégration d'abord régionale.}

47. De façon classique, plusieurs arguments sont avancés en faveur d'une intégration juridique d'abord régionale: tout d'abord la nécessité d'unification qui se ferait souvent plus pressante dans une région limitée, ensuite l'existence de points communs ou traditions communes plus nombreuses entre Etats d'une même région, enfin la taille raisonnable d'un groupe régional de négociations qui rendrait les projets plus réalistes et plus facilement adoptables.

- Une nécessité d'unification régionale souvent plus pressante. Les arguments économiques régionaux.

48. Les arguments économiques régionaux conservent une grande portée. Si l'on revient à l'historique de la Communauté européenne et à la déclaration Schumann, les objectifs apparaissent nettement : afin d'éviter de nouveaux conflits susceptibles de conduire à une guerre mondiale, les six premiers Etats, fondateurs de la CEE, se proposaient de créer une communauté économique au sein de laquelle les échanges seraient facilités. Libre circulation des marchandises, puis des capitaux et des personnes, ce sont bien les objectifs de créer une solidarité de fait d'abord en matière économique qui ont présidé à la création de la CEE. D'ailleurs, les échanges économiques entre pays voisins sont fréquents; partant ce de constat, l'idée de Jean Monnet d'officialiser et de faciliter ces échanges grâce à une structure communautaire allait se révéler visionnaire. L'exemple de l'Europe montre également que l'union économique, si elle est bien réalisée et s'il existe suffisamment de confiance entre les Etats membres, peut conduire à une Union politique (même si l'idée d'une Europe fédérale en effraie 
encore plus d'un). La reconnaissance de libertés au sein du marché intérieur a également conduit à des immixtions dans les procédures civiles nationales : la Cour de Justice des Communautés a à plusieurs reprises affirmé que les règles nationales de procédure ne devaient pas freiner voire bloquer la circulation juridique au sein de l'Union, ni défavoriser une partie étrangère (ressortissante d'un autre Etat membre), ${ }^{132}$ ce qui a conduit à une diminution des barrières procédurales au sein du marché intérieur. ${ }^{133}$ D'un point de départ purement économique, on est donc passé à la valorisation de la libre circulation des personnes et de l'idée de citoyenneté de l'Union, qui suppose une absence de discrimination notamment dans les procédure juridictionnelles.

- Davantage de points communs entre pays voisins.

49. Souvent sont évoqués les points communs ou les traditions juridiques communes plus grandes entre pays voisins, qui faciliteraient l'adoption d'un droit uniforme. L'exemple de la Communauté européenne montre que les diversités sont quand même toujours nombreuses et que les perceptions nationales sont souvent éloignées les unes des autres. Même si les Etats-Unis, lors des négociations dans le cadre de la Conférence de La Haye à propos du projet de convention mondiale sur les jugements, ont fait remarquer que la Convention de Bruxelles avait pu être adoptée en raison de traditions juridiques communes des Etats européens et que les Etats-Unis avaient une conception de la fonction sociale du pouvoir juridictionnel qui s'opposait fondamentalement à celle de la plupart des Etats membres de l'Union européenne, il n'empêche que l'unification régionale en matière de procédure civile est rendue difficile du fait que la matière est très liée à la souveraineté des Etats et souvent aussi à leur Constitution, qui contient un certain nombre de garanties et d'impératifs en matière d'organisation juridictionnelle et de procédure.

- La taille raisonnable du groupe de discussion et de préparation des projets

50. Une intégration régionale suppose tout d'abord des négociations poussées et une confiance raisonnable en la loyauté des membres participant au débat. L'Europe s'est faite tout d'abord à six (Allemagne, France, Italie,

132 H. Kronke, Ziel -Methoden, Kosten -Nutzen : Perspektiven der Privatrechtsharmonisierung nach 75 Jahren UNIDROIT, JZ2001, p. 1155.

133 CJCE, aff. C-43/95, Data Delecta/MSL Dynamics, Rec. 1996. I.4661, \$13; Hayes, Rec. 1997. I. 1711, \$13. 
Pays-Bas, Belgique et Luxembourg), et c'est ainsi qu'elle a pu créer des assises solides permettant au fil du temps des élargissements qui ont conduit aujourd'hui à une Union européenne de quinze Etats. La taille raisonnable du groupe permet souvent une synthèse plus rapide et des compromis de rédaction. Des questions se posent pour l'avenir au regard de l'élargissement projeté dans les prochaines années, et qui va faire entrer dans la Communauté européenne des Etats d'Europe centrale de culture économique et juridique en partie différentes de celles des membres actuels. La règle de l'unanimité au sein du Conseil, qui est encore en vigueur pour quelques années, ne facilite pas la prise de décision.

\section{Les effets d'une intégration régionale préalable}

- Effets positifs

51. Un des effets positifs essentiels d'une intégration régionale préalable est le rôle de "modèle" qu'elle peut jouer, de "moteur» en vue d'une extension de l'harmonisation sur le plan mondial. N'a-t-on pas bien souvent dit que la réussite exceptionnelle de la Convention de Bruxelles du 27 septembre 1968 en matière de compétence judiciaire, de reconnaissance et d'exécution, avait poussé les autres Etats du monde à rechercher dans le cadre de la Conférence de La Haye une certaine harmonisation de ces mêmes questions ? La réalisation à l'échelon régional montre la "faisabilité " du projet, certes sur une échelle réduite par rapport à celle du monde. Mais elle crée un facteur d'incitation qui peut jouer en faveur de l'adoption de conventions au champ géographique plus étendu. Les Etats membres d'une communauté régionale, riches d'une expérience commune d'unification, peuvent jouer un rôle central dans des négociations en vue d'une convention mondiale.

\section{- Effets pervers}

52. Des effets pervers sont particulièrement décelables au regard de l'évolution des compétences au sein de l'Union européenne. Il apparaît que la coopération judiciaire européenne en matière civile, telle qu'elle a été " communautarisée " par le Traité d'Amsterdam qui l'a fait passer du troisième au premier pilier, donnant ainsi compétence législative directe à la Communauté dans ce domaine, ${ }^{134}$ va rendre plus difficiles les négociations mondiales de conventions, comme par exemple celle projetée

134 "Abbau prozessualer Schranken im Binnenmarkt", v. B. HEb, Der 
dans le cadre de la Conférence de La Haye, ${ }^{135}$ ou encore les travaux d'UNIDROIT.136 Quelles seront les difficultés? Non seulement des questions de détermination de compétences, mais également de style et méthode de travail, de qualité pour reconnaître les problèmes et les résoudre, ${ }^{137}$ peut-être aussi des questions de pouvoir. ${ }^{138}$ En outre, la " communautarisation » de la procédure civile et du droit international privé détache ceux-ci des sources internationales classiques, ${ }^{139}$ l'Europe connaît à l'heure actuelle une régionalisation notable de son droit, ${ }^{140} \mathrm{ce}$ qui risque de l'isoler du reste du monde ou en tout cas de faire peser sur les Etats tiers et les institutions mondiales de négociation de traités une certaine pression. ${ }^{141}$

Binnenmarktprozeb, $J Z 1998$, p. 1023. Sur la fonction d'intégration de la procédure civile européenne, v. B. HEb, Die Integraitonsfunktion des europäischen Zivilverfahrensrechts, IPRax 2001, p. 389.

135 Sous la réserve contenue dans l'article 65 Traité CE que les mesures envisagées soient "nécessaires au bon fonctionnement du marché intérieur ", ce qui sera souvent le cas.

136 V. H. Schack, “Die EG-Kommission auf dem Holzweg von Amsterdam», ZEuP 1999 , p. 805.

137 V. H. Kronke, préc., JZ2001, p. 1155.

138 Comment par exemple ne serait-ce que définir clairement le champ d'application territorial de la future Convention mondiale sur les jugements par rapport au règlement CE dit Bruxelles I ?

139 En ce sens, H. Kronke, ibid. V. l'exemple que cite l'auteur : pendant trois ans, les gouvernements des Etats membres de l'Union européenne ont négocié avec le reste du monde, au sein d'UNIDROIT, la convention en matière de sûretés mobilières, alors que la Commission européenne ne s'est même pas rendue aux négociations auxquelles elle était conviée, puis quelques semaines avant la Conférence diplomatique, elle intervient et conteste aux Etats membres (sans doute d'ailleurs à juste titre) leur compétence pour négocier. Cette situation révèle un problème structurel qui risque de se reproduire et de rendre plus compliquée l'adoption de textes internationaux. C'est pourquoi il est réellement souhaitable que la Communauté européenne devienne membre à part entière des organisations mondiales au sein desquelles se négocient les traités.

140 V. B. HEb, Die « Europäisierung » des internationalen Zivilprozessrechts durch en Amsterdamer Vertrag -Chancen und Gefahren, NJW2000, p. 23 et spécialement p. 26, qui se pose la question de savoir " si et dans quelle mesure la coopération judiciaire de l'Union européenne doit se détacher de l'unification mondiale en matière civile $n$. Lauteur suggère une véritable discussion de politique juridique sur l'étendue des mesures à adopter sur le fondement de l'article $65^{\circ}$ Traité CE, ainsi que sur les rapports avec les autres instruments d'unification, mondiaux eux.

${ }^{141}$ V. B. HEb, «Die Integrationsfunktion des Europäischen Verfahrensrechts», IPRax 2001 , p. 395. 
53. Des questions à la fois techniques et constitutionnelles peuvent rendre difficile le passage d'une intégration régionale à une harmonisation mondiale de certains secteurs tels que ceux du droit international privé et de la procédure civile. Vaut-il mieux d'abord dégager sur le plan mondial des standards minimum qui pourront être enrichis et conduire à une unification plus poussée dans une entité territoriale régionale, ou bien convient-il de développer d'abord une construction régionale, forte de ses atouts tels que traditions communes des Etars membres, histoire partagée, valeurs communes etc ? N'oublions pas que malgré un "fonds commun " entre ces nations, il n'en subsiste pas moins des différences, et que le concept d'ordre public national n'est pas prêt de disparaitre. Ch. Kohler évoquait par exemple ${ }^{142}$ les risques constitutionnels qu'il existe à supprimer totalement la procédure d'exequatur dans l'Etat membre requis, en relevant que la Cour constitutionnelle allemande avait jugé en $1983^{143}$ qu'il existait des limites constitutionnelles à l'introduction d'une possibilité générale d'exécution directe des titres exécutoires étrangers en Allemagne ; une telle exécution ne serait possible que si les normes matérielles appliquées au soutien de la décision étrangère n'étaient pas contraires à l'ordre public allemand et si la procédure répondait au standard minimum de l'Etat de droit.

54. On peut se poser la question de savoir si le développement parallèle d'un droit international privé intereuropéen et d'un droit international impliquant des Etats tiers est judicieux à l'époque de la " globalisation économique " ${ }^{144}$ que nous connaissons. En effet, la Communauté européenne suit une évolution qui se caractérise par l'apparition d'un espace juridique de plus en plus soudé par des règles de droit international privé et de procédure civile unifiées. Une des tendances est traduite par l'utilisation fréquente du principe dit Herkunftslandprinzip, principe qui traduit la confiance envers l'Etat d'origine au sein de l'Union européenne, mais ne s'applique pas envers les Etats tiers. Une distance, voire un abîme se crée ainsi peu à peu entre un espace d'intégration juridique extrêmement

142 Comp. B. HEb, ibid., qui se pose la question de savoir si les Etats membres de l'Union européenne seront encore intéressés à financer des institutions internationales.

$143 \mathrm{Ch}$. Kohler, "Systemwechsel im europäischen Anerkennungsrecht : Von der EuGVVO zur Abschaffung des Exequaturs", in : Systemwechsel im europäischen Kollisionsrecht, Munich, Beck 2002, p. 158 et s.

144 C. const. féd., 22 mars 1983, BVerfGE 63, p. 343 et spéc. p. 366. 
poussée et le reste du monde. D'aucuns ${ }^{145}$ ont pu affirmer que c'était face à une tel paysage juridique que les Etats-Unis avaient été à l'origine du projet de Convention mondiale sur les jugements débattu à La Haye. Force est de constater que régionalisation et globalisation se font face, ce qui soulève les difficultés déjà énoncées plus haut.

\section{Les effets d'une intégration mondiale sur l'intégration régionale. Un possible parallélisme des démarches?}

55. On pourrait penser que l'existence préalable de conventions internationales au champ élargi permettrait aux entités régionales de les intégrer dans les rapports entre leurs Etats membres et contribuerait ainsi à une harmonisation encore plus grande des domaines juridiques concernés. Et pourtant, la communautarisation du droit international privé et de la procédure civile se fait parfois au détriment des traités internationaux. Un exemple peut être cité pour illustrer cet état de fait : les règles du règlement $\mathrm{CE} \mathrm{N}^{\circ} 1347 / 2000$ relative à la compétence, la reconnaissance et l'exécution des décisions en matière matrimoniale et en matière de responsabilité parentale font apparaître quelques contradictions avec la Convention de La Haye sur la protection des mineurs, qui aborde elle aussi la question de l'autorité parentale sur les enfants mineurs. ${ }^{146}$ L'existence préalable de conventions mondiales pourrait inciter les Etats membres d'organisations régionales à étendre à leurs rapports internes les stipulations de ces textes. Mais il apparaît que souvent, l'intégration régionale a des ambitions plus grandes et espère aller plus loin dans l'unification qu'une convention mondiale à standards minimum. C'est ainsi que des groupements divers légiferent, parfois simultanément ou presque, sans souci suffisant de coordination.

56. Aujourd'hui, il apparaît impossible que les deux niveaux d'harmonisation (à défaut d'unification, en tout cas sur le plan mondial) continuent à s'ignorer. Ainsi, la Conférence de La Haye de droit international privé devrait, nous semble-t-il - et c'est d'ailleurs la direction

145 V. Vorwort (avant-propos) de Systemwechsel im europäischen Kollisionsrecht, Munich, Beck éd., 2002, par J. Baur et H-P. Mansel.

146 V. par ex. H.- P. Mansel, «Zum Systemwechsel im europäischen Kollisionsrecht nach Amsterdam und Maastricht", in : Systemwechsel im europäischen Kollisionsrecht, op. cit., p. 14. 
qu'elle semble prendre - accepter et les clauses de "déconnexion" permettant au droit international privé communautaire de garder ses effets et de ne pas être atteint, dans les rapports entre Etats membres, par l'adoption d'une Convention mondiale, et l'adhésion de la Communauté européenne $\mathrm{e}^{167}$ afin que celle-ci puisse participer directement aux négociations et exercer sa compétence externe. Les deux entités de production de normes doivent tenir compte l'une de l'autre afin que soit écarté le danger que perçoit un auteur ${ }^{148}$ de voir à la longue toutes les forces de l'Union européenne et des Etats membres être épuisées par la construction d'une justice purement européenne, ce qui conduirait à un désert d'harmonisation sur le plan mondial. ${ }^{149}$ La Conférence de La Haye pourra jouer un rôle essentiel de rapprochement par exemple entre l'Union européenne et les Etats de l'Europe de l'Est ou de la Méditerranée. ${ }^{150}$ Il est évident que les actions doivent être concertées afin d'éviter des cacophonies et contrariétés ${ }^{151}$ qui ne faciliteraient ni le libre accès à la justice ni la création d'un espace judiciaire harmonisé. La globalisation en marche suppose la création de liens d'information et de coopération entre

147 V. Systemwechsel im europäischen Kollisisonsrecht, op.cit., Diskussionsbericht p. 206 (remarque de K. Boele-Woelki, qui estime également qu'une unification du droit successoral international au sein de l'Union européenne pourrait avoir lieu par ratification par les Etats membres de la Convention de La Haye de 1989 sur le droit des succesions. D'autres concurrences peuvent être relevées entre l'Union européenne, ses projets, et les conventions de La Haye déjà existantes, par exemple en matière de preuve. On peut se demander si une telle ratification générale ne pourrait pas être le fait des institutions communautaires elles-mêmes, ce qui unifierait plus rapidement et en même temps le droit international applicable tant entre les Etats membres qu'entre eux et des Etats tiers.

148 Sur cette question, v. notamment H. Van Loon, "The Hague Conference on Private International Law in a time of Globalisation : New Challenges and New Conventions in the fields of Civil Procedure and Applicable Law", in Systemwechsel im europäischen Kollisionsrecht, op. cit., p. 193 et s.

149 H. Duintjer Tebbens, préc., in Systemwechsel im europäischen Kollisionsrecht, op. cit., p. 192.

150 Sur le rôle de la Conférence de La Haye de droir international privé, v. H. Van Loon, préc ; p. 197 : elle devrait être " an open organisation : open both in terms of its receptivity towards the potential contribution of a multitude of traditions on its policies, and also in terms of its outlook on the world as an increasingly interdependent system of an increasingly diverse variety of actors, not limited to the traditional nation State, representing different, sometimes conflicting, traditions ".

151 En ce sens, H. Van Loon, ibid. 
les différents systèmes juridiques; de tels réseaux de communication et d'information sont extrêmement souhaitables et nécessaires, peut-être plus qu'une unification qui impliquerait des changements juridiques majeurs dans les systèmes juridiques de certains Etats qui s'y résoudraient sans doute difficilement. Il est en outre sans doute plus facile d' «unifier » ou d' "harmoniser " dans des domaines neufs, par exemple celui des techniques nouvelles du commerce électronique, qui ne sont pas encore ancrées dans des traditions étatiques nationales profondes. ${ }^{152}$ Les projets encore plus ambitieux, tel que celui de Convention mondiale sur la compétence et les jugements, requièrent une souplesse considérable pour leur adoption, et un contrôle indispensable de la prévisibilité des règles qu'ils édictent. En effet, ces traités mondiaux sont destinés à faciliter l'accès à la justice des citoyens, ce qui suppose clarté des règles et prévisibilité de leur application et interprétation. La flexibilité ${ }^{153}$ est donc bien l'un des maîtres mots de projets ambitieux, comme l'illustre par exemple la Convention de $\mathrm{La}$ Haye du 25 octobre 1989 en matière d'enlèvement international.

\section{Conclusion}

57. A l'heure actuelle, le phénomène de " communautarisation " engendré par le Traité d'Amsterdam fait naître des risques au regard des tentatives d'harmonisation en matière de procédure civile engagées sur le plan mondial. L'Union européenne doit éviter de se renfermer dans des règles purement communautaires qui, si elles sont nécessaires à l'achèvement d'un espace judiciaire européen et à l'accès à la justice du citoyen de l'Union, ne doivent pas mettre en danger les recherches d'harmonisation à une échelle plus grande. Une concertation et une coopération doivent donc avoir lieu entre les institutions communautaires et les grandes organisations internationales impliquées dans l'unification du droit. Il reste à espérer que la Commission européenne fera sienne cette analyse et procédera à de nombreuses consultations et échanges de vues avant de presser le Conseil à légiférer de façon parfois hâtive et donc insuffisamment réfléchie. Il est à prévoir que des processus d'harmonisation et d'autres d'unification

152 V. A. Malan, La concurrence des conventions d'unification des règles de conflit de lois, PUF Marseille, 2002.

153 Dans le même sens, v. H. Van Loon, préc., p. 198. 
continueront à se dérouler en parallèle à des vitesses variables, et au sein d'organisations diverses (Conférence de La Haye, UNIDROIT, UNCITRAL, Communautés régionales telles que la Communauté européenne...), avec des influences réciproques. ${ }^{154}$

154 En ce sens, v. P. Biavati, «Is Flexibility a Way to the Harmonization of Civil Procedural Law in Europe ?", in: Essays on Transnational and Comparative Civil Procedure, sous la dir. De F. Carpi et M.A. Lupoi, Turin, Giappichelli éd., 2001, p. 85 et notamment p. 96 et s.

155 Dans le même sens, G. Walter et F. Walther, International Litigation : Past experiences and Future Perspectives, in Essays on Transnational and Comparative Civil Procedure, sous la dir. de F. Carpi et M.A. Lupoi, Turin, Giappichelli éd., 2001, p. 155 et notamment p. 198. Ces auteurs pensent que " the situation of tomorrow will also resemble the situaiton of today: International or transnational civil procedure will be a patchwork of all kinds of provisions". 